\title{
Die Organisationsreform der SPD 2017-2019: Jung, weiblich und digital?
}

\author{
Dennis Michels $\mathbb{D} \cdot$ Isabelle Borucki $\mathbb{D}$
}

Eingegangen: 20. Dezember 2019 / Überarbeitet: 3. August 2020 / Angenommen: 4. August 2020 /

Online publiziert: 3. September 2020

(C) Der/die Autor(en) 2020

Zusammenfassung Die Organisationsreform der SPD 2017-2019 beinhaltete die Einführung digitaler Onlinethemenforen, die mit Antrags- und Rederecht für Bundesparteitage ausgestattet wurden. Der Beitrag beantwortet die Frage, warum die Reformmaßnahme durchgeführt wurde, indem legitimatorische Begründungsmuster der Parteiführung als kausaler Mechanismus des Reformprozesses herausgearbeitet werden. Per interpretativem Process Tracing wird zur Theoriebildung des (digitalen) Parteienwandels beigetragen. Es wird aufgezeigt, dass neue Onlinethemenforen auf eine Basisbeteiligung in der Willensbildung abzielen. Begründet werden die Reformen mit der Niederlage bei der Bundestagswahl 2017, einer veränderten gesellschaftlichen Erwartungshaltung an Möglichkeiten digitaler Beteiligung sowie der Bindung von jungen Neumitgliedern, die traditionellen Parteistrukturen ablehnend gegenüberstehen. Weiterhin sollen Onlinethemenforen eine ortsunabhängige, themenbezogene Vernetzung der Mitglieder ermöglichen und eine eigene Informationspolitik innerhalb der Partei etablieren, neben Massenmedien und sozialen Medien. Zudem wird die Effizienz der Parteiarbeit als weiteres Begründungsmuster angeführt.

Schlüsselwörter Digitalisierung $\cdot$ Kommunikation · Beteiligung ·

Parteiorganisation $\cdot$ Reformgründe

Dennis Michels ist wissenschaftlicher Mitarbeiter, Dr. Isabelle Borucki ist Nachwuchsgruppenleiterin, beide im Forschungsprojekt „Digitale Parteienforschung (DIPART) - Parteien im digitalen Wandel“ an der NRW School of Governance der Universität Duisburg-Essen. Das Forschungsprojekt wird im Rahmen des Verbundprojekts „,Digitale Gesellschaft“ durch das Ministerium für Kultur und Wissenschaft des Landes Nordrhein-Westfalen gefördert.

D. Michels $(\square) \cdot$ I. Borucki

NRW School of Governance, Universität Duisburg-Essen, Campus Duisburg, LS 123,

Lotharstraße 53, 47057 Duisburg, Deutschland

E-Mail: dennis.michels@uni-due.de 


\title{
The Organizational Reform of the German Social Democratic Party (SPD) from 2017-2019: Young, Female, and Digital?
}

\begin{abstract}
The organizational reform of the SPD from 2017-2019 included the introduction of online topic forums, which contain a right for its members to make proposals and to speak at party conferences. This article answers the question of why the reform was conducted by uncovering legitimating reasons brought forward by the party leadership and framing them as a causal mechanism of the reform. By using an interpretive process tracing approach, the article contributes to theory building of (digital) party change. It is shown that new online topic forums aim at improved participation possibilities for the party base in decision-making processes. The reasons given for the reform are the defeat in the 2017 federal elections, a change in society's expectations towards possibilities of digital participation, and engaging young new members who are hostile to traditional party structures. In addition, the so-called online topic forums allow for a location-independent, topic-related networking of members and the possibility of establishing a separate information policy within the party, alongside mass and social media. Moreover, the efficiency of party work is cited as the final justification pattern.
\end{abstract}

Keywords Digitalization - Communication · Participation · Party organization · Reasons for reform

\section{Einleitung}

Digitalisierung als geradezu gehäusesprengender Transformationsprozess macht auch vor etablierten Institutionen wie politischen Parteien nicht halt. Im Gegenteil sind gerade Parteien in ihrer internen Verfasstheit von zwei Seiten unter Reformdruck geraten: einerseits vonseiten ihrer Wähler*innen und Unterstützer*innen, die mehr Transparenz, Responsivität und Information von Parteien einfordern, andererseits vonseiten ihrer Mitglieder, die sich mehr innerparteiliche Partizipation wünschen und mit diesen Ansprüchen an die Parteiführungen herantreten. Insbesondere digitale Kommunikationsinstrumente schüren Hoffnungen auf neue Möglichkeiten demokratischen Austauschs zwischen Parteien und ihren Unterstützer*innen (Jun 2009, S. 203).

Diesem Spagat sieht sich insbesondere die SPD ausgesetzt, die mit sinkenden Wähler*innenzahlen und einer Veränderung ihrer Mitgliederstruktur (Niedermayer 2019) konfrontiert ist. Die Partei diskutiert seit Ende 2017 im Angesicht des gesellschaftlichen Wandels verstärkt über digitale Kommunikations- und Beteiligungsinstrumente in der Parteiarbeit. Schließlich wurde auf dem Parteitag im Dezember 2019 die Einführung von Onlinethemenforen beschlossen, die als digitales Herzstück des Reformprozesses \#SPDerneuern 2017-2019 gelten. Zwar ist eine Verbindung der Reform zur Wahlniederlage 2017 und zum allgemeinen digitalen Wandel scheinbar offenkundig. Wie die Lage der Partei sich allerdings für die Parteiführung darstellte und welche Gründe sie letztlich dazu brachten, sich für die Schaffung der 
neuen, digitalen Parteistruktur mit Antrags- und Rederecht auf Bundesparteitagen $\mathrm{zu}$ entscheiden, ist bislang unbekannt.

Vor diesem Hintergrund erforscht der Beitrag Begründungsmuster und subjektive Handlungslogiken der Parteiführung als kausale Mechanismen bei der Einführung der Onlinethemenforen. Er beantwortet die Frage, warum Onlinethemenforen im Rahmen des Reformprozesses \#SPDerneuern 2017-2019 eingeführt wurden. Hierdurch trägt der Beitrag dazu bei, die „Blackbox“ innerparteilicher Entscheidungsprozesse zu öffnen und dabei insbesondere die Parteiführung als „Konstrukteurin“ von Parteireformen in den Blick zu nehmen. Ziel ist, Begründungsmuster herauszuarbeiten, die innerhalb des Entscheidungsprozesses zur Parteireform als zentraler legitimatorischer Baustein verstanden werden. Der zugrunde liegende Mechanismus der Reform wird über die Verknüpfung von Begründungsmustern mit Zielvorstellungen der Reform gebildet. Dieser Mechanismus entscheidet über die inhaltliche Ausrichtung, aber auch die Durchsetzung der Reform. Letztlich wird durch ihn über verschiedene innerparteiliche Aushandlungsrunden die Durchsetzung einer Reform der Gesamtpartei als legitim und wünschenswert vermittelt.

Die Forschungsfrage adressiert die performative Rolle von Reformtreibern und schließt damit an die Party-Change-Forschung an (Gauja 2017; Wiesendahl 2010b; Harmel und Janda 1994). Der in diesem Beitrag verwendete Forschungsansatz interpretativer Politikforschung (Nullmeier 2019) folgt dabei der Erkenntnis, dass die Wahrnehmung und Verarbeitung der Parteiumwelt durch die Parteiakteure eine zentrale Bedeutung bei der Erklärung von Parteireformen spielen (Gauja 2017, S. 14; Wiesendahl 2010a, S. 51; Bukow 2013b, S. 233-234) und zudem der innerparteiliche Entscheidungsprozess in der Parteiorganisation stärker in den Blick rücken sollte (Wiesendahl 2010a, S. 46). ${ }^{1}$

Folglich wird ein interpretativer Forschungszugang gewählt und eine fallbasierte Erklärungsstrategie im Rahmen einer qualitativen Einzelfallstudie verfolgt (Gläser und Laudel 2010). Die SPD wurde als Fall ausgewählt, da der allen Parteien zugeschriebene Strukturkonservatismus (Bukow 2013a, S. 13) bei ihren letzten Reformrunden besonders deutlich zutage trat (Jun 2018), was eine besondere Bewährungsprobe für die untersuchten Mechanismen darstellt. Zudem sind die von ihr eingeführten und nun in den Statuten festgeschriebenen Onlinethemenforen eine einzigartige Innovation digitaler Parteireformen. Dies macht neuartige Begründungsmuster erwartbar. ${ }^{2}$

Auf Basis von acht teilstrukturierten Expert*inneninterviews mit Mitgliedern und Mitarbeiter*innen der Parteiführung ergänzt durch Beschlussdokumente des Parteivorstands und des Parteitags wurde daher eine interpretative Prozessrekonstruktion (Nullmeier 2019; Beach und Pedersen 2019; Collier 2011) durchgeführt. Über einen doppelten Codierprozess wurden sechs verschiedene Begründungsmuster der digitalen Parteireform identifiziert.

Im Folgenden werden zunächst Parteireformen näher spezifiziert und vor dem Hintergrund des politischen Formwandels in der digitalen Konstellation betrachtet.

\footnotetext{
1 Der Ansatz wird im Analysezugang (Abschnitt „Analysezugang: Die Parteienführung als ,Konstrukteurin' der Parteireform“) näher erläutert.

2 Eine detaillierte Fallbegründung erfolgt im Abschnitt „Fallauswahl und Verallgemeinerungspotenzial“.
} 
Nach der Darstellung des Literaturstands zu Reformgründen werden digitale Reformen als neue Kategorie von Reformen dargestellt. Anschließend wird der Analysezugang erläutert, der eine Konstruktion der Reform durch die Parteiakteure annimmt. Nach einer Darlegung des methodischen Vorgehens werden die sechs identifizierten Begründungsmuster für die Einführung der Onlinethemenforen als Ausdruck des digitalen Charakters von Parteistrukturen geschildert. Abschließend werden Implikationen im Zusammenhang mit bestehendem Wissen um Parteireformen und der Einführung digitaler Technologie in Parteien diskutiert.

\section{Parteireformen und Digitalisierung - warum reformieren sich Parteien digital?}

\subsection{Warum reformieren sich Parteien?}

Zur Spezifikation von Parteireformen greift dieser Beitrag zunächst auf die Definition nach Jun (2009, S. 188) zurück. Er versteht darunter ,alle planvollen, auf strategische Entscheidungen zurückzuführenden Veränderungen der Organisationsstrukturen einer Partei, die unmittelbar das Binnenleben betreffen“. Enger gefasst werden mit Bukow (2009, S. 213) besonders solche Veränderungen der formalen internen Strukturen als Parteireformen verstanden, die in der Parteisatzung festgeschrieben werden. ${ }^{3}$

Gründe für Parteireformen werden mit Gauja (2017, S. 8-10) auf den Ebenen des politischen Systems, des Parteiensystems und der innerparteilichen Interaktionen identifiziert. Sie betont, dass Gründe, die im politischen System verortet werden, langfristiger zu denken sind als beispielsweise Wahlergebnisse, die in der Wahrnehmung der Parteiakteure eine eher situative Wirkung entfalten. Mit innerparteilichen Gründen für Parteireformen beschreibt sie insbesondere machtstrategische Gründe der Parteieliten. Als Gründe für Parteireformen gelten nach Gauja (2017, S. 9):

- gesellschaftliche Erwartungen an „,moderne“ oder „,demokratische“ Politik: Grund für eine Reform wäre beispielsweise, veränderten Erwartungen an Beteiligungsmöglichkeiten, an spezifische Interaktion mit den Massenmedien oder die Nutzung „moderner“ Technologie in einer Partei zu entsprechen. Auch die Anpassung an weitere gesellschaftliche Trends und den aktuellen Zeitgeist fällt hierunter.

- Parteienwettbewerb und Wahlen: Insbesondere nach Wahlniederlagen sind häufig Parteireformen zu beobachten. So können Ziele wie eine verbesserte Außendarstellung, Rückgewinn von Vertrauen und Legitimation, aber auch das Kopieren von Erfolgsmodellen anderer Parteien Gründe für Parteireformen sein.

- Innerparteiliche Gründe: Darunter fallen sowohl das Ziel einer Erhöhung der Mitgliederzahlen der Partei als auch machstrategische Gründe, wie die Ausweitung von Einfluss des Parteivorstands oder aber die stärkere Beteiligung der Basis auf Kosten der mittleren Parteifunktionäre.

\footnotetext{
3 Programmarbeit und Personalentscheidungen sind somit nicht Teil der hier gemeinten Reformen, auch wenn sie z. B. bei Gauja (2017, S. 17) oder Walter-Rogg (2013) als programmatische und personelle „Reformen" bezeichnet werden.
} 
Allerdings bezieht sich Gauja (2017) mit ihrem Erklärungsansatz nicht auf die Einführung digitaler Parteistrukturen, denen revolutionäres Potenzial in Bezug auf einen Wandel von Organisationsstrukturen zugeschrieben wird (Chadwick und Stromer-Galley 2016; Bennett und Segerberg 2012). Die radikale Neuheit digitaler Organisationsstrukturen lässt veränderte Begründungsmuster für darauf bezogene Parteireformen erwarten. So wird im Folgenden auf den Formwandel von Parteien in der digitalen Konstellation eingegangen, um darauf aufbauend darzulegen, warum die hier betrachtete Reform einer neuen Kategorie der ,digitalen Parteireform“ zugeordnet wird.

\subsection{Formwandel von Parteien in der digitalen Konstellation}

Zunächst ist für den hier untersuchten Fall der Parteiorganisationreform festzuhalten, dass es sich um einen Wandlungsprozess entlang der digitalen Transformation handelt. Berg et al. (2020) schlagen drei analytische Ebenen vor, auf denen Digitalisierung eine Bedeutung für Politik in der sogenannten ,digitalen Konstellation“ entfaltet: Eigenschaften digitaler Technik, praktisch realisierte Affordanzen ${ }^{4}$ und der politische Formwandel. Aktuell prägen digitale Technologie Eigenschaften wie „Archivierbarkeit, Vernetzbarkeit und Prozessierbarkeit“" (Berg et al. 2020, S. 14) digitaler Daten. Dies führt praktisch zur Affordanz der Many-to-many-Kommunikation, die jederzeit ,in Echtzeit“ möglich ist. Im Vergleich zur Logik politischer Kommunikation in den Massenmedien (Altheide und Snow 1979; Couldry 2008) scheint digitale Kommunikation schneller, direkter, interaktiver und multimodaler zu verlaufen (Dohle et al. 2014; Tenscher und Borucki 2015). Als weitere Affordanzen wurden mittlerweile die ortsungebundene Interaktion oder das Austauschen und gemeinschaftliche Zusammentragen von Daten über Datenbanken identifiziert (z. B. Profilseiten in sozialen Netzwerken, Wissensplattformen wie Wikipedia; Schmidt 2009).

Der auf digitalen Affordanzen aufbauende politische Formwandel äußert sich in Bezug auf politische Organisationen etwa in interaktiver Außenkommunikation oder horizontalen Organisationsweisen der ,connective action“ (Bennett und Segerberg 2012). Darüber hinaus zielen Organisationen im kommunikativen Bereich zunehmend darauf ab, ,virale“ Nachrichten zu verbreiten, um Aufmerksamkeit zu erlangen. Dafür stellen sie neue Mitarbeiter*innen ein und entwickeln neue Kommunikationsstandards (Dohle et al. 2014, S. 421-422). Zu beobachten ist ein Prozess, der zu einer Veränderung einer Organisationskultur, der Denkschemata der Organisationsmitglieder sowie ihrer zugrunde liegenden Fähigkeiten führt (Mergel et al. 2019, S. 12).

Der digital induzierte Formwandel äußerte sich in den deutschen Parteien bislang in Experimenten mit virtuellen Parteigliederungen oder direkten Kommunikationskanälen zwischen Parteiführung und Parteibasis, „Mitmachwahlkämpfen“, kollaborativer Programmarbeit oder ,virtuellen“ Mitgliedschaften (Bieber 2014; Marschall

\footnotetext{
4 Als Affordanz bezeichnen Berg et al. (2020, S. 14) eine „Beziehungsstruktur zwischen einem technischen Artefakt und dessen Nutzer*in [...], welche in einer konkreten Situation potenzielle Handlungsergebnisse ermöglicht oder beschränkt“.
} 
2013; Hanel und Marschall 2014; Gerl et al. 2016). Für die digital gewandelte Binnen- und Außenkommunikation scheint sich ein Professionalisierungstrend zu verstetigen. Dagegen waren Experimente mit Mitgliedern, die in digitalen Gremien Positionen erarbeiten oder Repräsentanten wählen, bislang von kurzer Dauer und aufgrund strenger Einhegung durch die Parteiführung von geringer Wirkung (Hanel und Marschall 2014, S. 200).

Als zentrale Herausforderung digitaler Experimente galt bislang, dass die Parteien am territorial geprägten Delegiertenprinzip strukturkonservativ festhielten. Versuche zur Ausschöpfung der Möglichkeiten digitalen Engagements verpufften somit besonders aufgrund eines Unwillens der Parteifunktionäre, ihnen eine dauerhafte Wirkmächtigkeit innerhalb der Entscheidungsstruktur zuzugestehen (Hanel und Marschall 2014, S. 200; Klecha und Hensel 2013, S. 134). Auch die Piratenpartei bildete hier keine Ausnahme: Massenkommunikation fand zwar im Internet statt, kollektive Entscheidungen wurden allerdings klassisch über Anträge an Parteitage organisiert (Klecha und Hensel 2013, S. 39-41). Das internetbasierte Diskussionsund Abstimmungsinstrument „Liquid Feedback“ war innerparteilich hoch umstritten und kam nicht über den Experimentalbetrieb hinaus (Klecha und Hensel 2013, S. 68-70). Die Plattform enttäuschte durch geringe Nachfrage und eine Verzerrung des Einflusses zur „Zeitelite“ - jenen, die Zeit im Überfluss hatten und die den dort produzierten Informationsüberschuss am besten bewältigen konnten (Koschmieder 2016; Klecha und Hensel 2013).

Die von der SPD im Rahmen der Parteireform „\#SPDerneuern“ eingeführten Onlinethemenforen sind vor diesem Hintergrund beachtenswert. Als vollständig digitales Gremium der Partei haben sie das Recht, Anträge an den Bundesparteitag zu stellen und beratende Delegierte dorthin zu entsenden. Damit erweitern sie den kollektiven Entscheidungsprozess in den digitalen Raum und begründen eine neuartige Ausprägung der Partei. Die zur Einführung einer solchen Organisationsstruktur notwendige Änderung der Parteistatuten wird hier als ,digitale Reform“ bezeichnet und von den bislang umgesetzten, ,,analogen“ Reformen abgegrenzt.

\subsection{Analoge und digitale Reformen}

Da Parteisatzungen unterschiedliche Regeln des Organisationsaufbaus und der kollektiven Entscheidungsfindung beinhalten, ist es sinnvoll, sich die verschiedenen Bereiche vor Augen zu führen, die statuarische Parteireformen betreffen können. Reformbereiche sind unter anderem Mitgliedschaftsformen und Mitgliederrechte (ordentliche Mitgliedschaft, Probemitgliedschaften, Rede- und Wahlrechte in Gremien), Gremien und Gliederungen (Schaffung/Auflösung, Größe, Zusammensetzung), Wahlordnung und Entscheidungsregeln innerhalb von Gremien (Wahlgrundsätze, Mehrheitsregeln, Fristen) oder die Parteihierarchie (Anordnung der Gliederungen und Gremien zueinander, Repräsentationsregeln - teils nur über das Parteiengesetz änderbar).

Die Beschaffenheit digitaler Technologie (Abschnitt „Formwandel von Parteien in der digitalen Konstellation“) macht eine Vielzahl digitaler Reformen denkbar (vgl. auch Bieber 2014, S. 176-177): Digitale Mitgliedschaftsformen, digitale Gliederungen oder digitale Gremien ohne territoriale Bindung könnten parallel zur analogen, 
Tab. 1 Unterschiede zwischen analogen und digitalen Gremien/Gliederungen. (Eigene Darstellung)

\begin{tabular}{lll}
\hline & $\begin{array}{l}\text { Analoge Gremien/Gliederungen } \\
\text { (z. B. Orts-, Kreis-, Landesverbän- } \\
\text { de; Arbeitsgemeinschaften) }\end{array}$ & $\begin{array}{l}\text { Digitale Gremien/Gliederungen } \\
\text { (z.B. Onlinethemenforen) }\end{array}$ \\
\hline $\begin{array}{l}\text { Zusammen- } \\
\text { setzung }\end{array}$ & Wohnortsprinzip & Wohnorts- oder Themenprinzip \\
$\begin{array}{l}\text { Interaktions- } \\
\text { zeit }\end{array}$ & $\begin{array}{l}\text { Synchron, punktuell } \\
\text { (feste Termine, Abarbeiten der } \\
\text { Tagesordnung) }\end{array}$ & $\begin{array}{l}\text { Synchron oder asynchron, permanent } \\
\text { (Speicherung in Datenbanken mit universeller Zu- } \\
\text { griffsmöglichkeit; Foren- oder Blogstruktur, Video- } \\
\text { konferenzen) } \\
\text { One-to-one, One-to-many, Many-to-many } \\
\text { Kommunika- } \\
\text { tionswege }\end{array}$ \\
$\begin{array}{l}\text { One-to-one, One-to-many } \\
\text { (moderierte Gruppengespräche, } \\
\text { begrenzte Sprechzeiten, Zwiege- } \\
\text { spräche) }\end{array}$ & $\begin{array}{l}\text { Direktnachrichten, Chaträume) } \\
\end{array}$ \\
\hline
\end{tabular}

ortsgebundenen Struktur entstehen. Ein digitales Rede- oder Wahlrecht (in analogen oder digitalen Gremien) könnte zu Formen der ,blended participation“ (Bieber 2014, S. 175; vgl. auch Kersting 2014), d. h. hybrider Beteiligung, führen, die durch eine Mischung von Elementen politischer Beteiligung gekennzeichnet sind, die je nach Bedarf digital oder analog organisiert werden. Die große Bandbreite von Möglichkeiten der Reform auf Basis der digital induzierten Affordanzen rechtfertigt dabei die Einführung der neuen Kategorie digitale Reformen. Möglich ist sowohl die Ersetzung analoger Strukturen durch digitale Strukturen als auch ihre Ergänzung zu einer Hybridform oder die Schaffung völlig neuer digitaler Strukturen mit neuartiger Funktion in Bezug auf die Organisation oder den kollektiven Entscheidungsprozess der Partei, was dann eine Innovation schüfe. Mischformen und Hybridreformen wären dementsprechend als Ergebnis einer digitalen Reform zu verstehen, die aus Teilen beider idealtypisch gedachter Strukturtypen ,,analog“ und „digital“ bestehen (Tab. 1).

In Abgrenzung zu den bisherigen digitalen Experimenten der deutschen Parteien (Hanel und Marschall 2014; Bieber 2014) stellen digitale Reformen einen auf Dauerhaftigkeit angelegten Formwandel der Partei dar. Eine solche Veränderung wird im Parteistatut festgeschrieben, da sie Regeln der kollektiven Entscheidungsfindung der Partei betrifft. Eine digitale Reform ist also an ihrer statuarischen Verfassung zu erkennen, wodurch sie über die bislang beobachtbaren neuen Organisations- und Kommunikationsinstrumente weit hinaus reicht. Als digitale Reform werden daher alle Reformen bezeichnet, die auf digitaler Technologie fußende Affordanzen in Parteistrukturen überführen, die im Parteistatut festgeschrieben werden.

\subsection{Die Onlinethemenforen der SPD als rein digitales Gremium}

Onlinethemenforen sind ein rein digitales Gremium, das den kollektiven Entscheidungsprozess der Partei in den digitalen Raum erweitert. Der Aufbau einer neuen Entscheidungsstruktur mittels der Onlinethemenforen hat somit parallel zur bestehenden Entscheidungsstruktur eine Doppelläufigkeit ergeben: Onlinethemenforen sind ebenso wie territorial basierte Gliederungen und Gremien berechtigt, Anträge an den Bundesparteitag zu stellen und Delegierte zu entsenden. Ebenso wie die Ar- 
beitsgemeinschaften der SPD erhalten die Onlinethemenforen zwei Delegierte mit Rederecht, aber ohne Stimmrecht auf dem Bundesparteitag.

Onlinethemenforen fördern die themenzentrierte Zusammenarbeit von Parteimitgliedern und umgehen letztlich das bislang geltende Territorialitätsgesetz, das die Zusammensetzung von Parteigliederungen nach Wohnortsprinzip statt thematischen Interesses festschreibt. Zudem bauen die hier untersuchten Themenforen auf Mittel der digitalen Kommunikation zur innerparteilichen Abstimmung. Zwar stehen (Stand Juli 2020) die innere Organisation und Entscheidungsstruktur der Onlinethemenforen noch nicht fest, allerdings kann davon ausgegangen werden, dass diese die neuen Möglichkeiten der Asynchronität, direkter Kommunikationsmöglichkeit oder Many-to-many-Kommunikation zur Erarbeitung gemeinsamer Positionen und Dokumente nutzbar machen werden.

Fraglich ist im Angesicht dieser Neuheiten, warum Parteien ihren digitalen Formwandel vorantreiben und digitale Reformen implementieren. Im Folgenden wird der Analysezugang dargestellt, der der Rekonstruktion von Gründen für die digitale Parteireform der SPD unterliegt.

\section{Analysestrategie und methodisches Vorgehen}

\subsection{Analysezugang: Die Parteiführung als „Konstrukteurin“ der Parteireform}

Ausgangspunkt der Analyse ist die Konstruktion der Parteireform durch die Parteiakteure. Im Kern geht es darum, dass nicht objektive Gründe ,gemessen“ werden, sondern subjektive Begründungen der Parteiführung rekonstruiert werden. Die von der Parteiführung bei der Initiierung der Reform vorgebrachten Begründungen werden über den kollektiven Entscheidungsprozess der Partei zu den Gründen der Gesamtpartei, eine Reform durchzuführen. Entscheidend ist, dass die Gründe für Reformen nicht als „objektive“ und „messbare“ Treiber von Reformen verstanden werden sollten, sondern konstruktivistisch gewendet als wahrgenommene Realität der Parteiakteure, die sie als Rationalisierung und Legitimierung ihrer Handlungen argumentativ vorbringen (Gauja 2017, S. 13-15; Deschouwer 1992, S. 17). Ging man lange Zeit von einer rationalen Reaktion der Akteure auf „Impulse“ oder „Schocks“ aus der Umwelt aus (Gauja 2017, S. 14; Harmel und Janda 1994; Panebianco 1988), so spricht Wiesendahl (2010a, S. 51) von einer „Gewahrwerdung, Deutung und Verarbeitung" der äußeren Einflüsse durch die Parteiakteure (vgl. auch Bukow 2013b, S. 233-234). Dadurch werden die Perzeptionen der beteiligten Akteure zum Ansatzpunkt, wenn es darum geht, den Ablauf von Reformprozessen in der Partei abzubilden. Die Aussagen der „Konstrukteur*innen“ der digitalen Organisationsreform werden zum zentralen Untersuchungsgegenstand, da aus ihnen Begründungsmuster interpretiert werden, die die Auswirkungen der Reform auf die Struktur der Partei bestimmen. Die Begründungsmuster werden als kausaler Mechanismus von (digitalen) Parteireformen verstanden (Abb. 1). Sie dienen der legitimatorischen Durchsetzung der Reform von der Initiative bis hin zum Reformbeschluss auf dem Parteitag. 


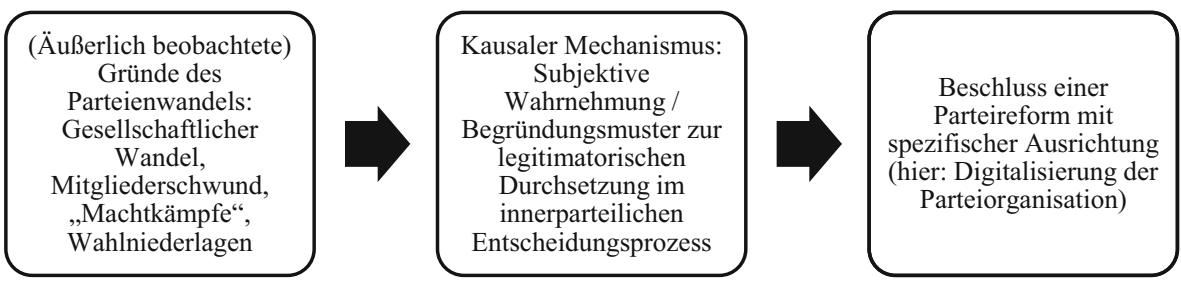

Abb. 1 Begründungsmuster zur legitimatorischen Durchsetzung der Reform als kausaler Mechanismus. (Eigene Darstellung)

Es bleibt zu klären, wie aus den Begründungen und Motiven einzelner Parteiakteure (auf der Mikroebene) eine neue Parteistruktur (auf der Mesoebene) erwächst. So wird der Annahme gefolgt, dass Vorgänge auf der Ebene der Gesamtpartei erklärbar sind durch Vorgänge auf der Ebene der Parteiakteure (Nullmeier 2019, S. 164). Innerhalb der verschiedenen Akteursgruppen der Partei ist es die Parteiführung, die Reformen anstößt und in der Lage ist, sie durchzusetzen (Bukow und Poguntke 2013, S. 196). Vor dem Hintergrund ihrer subjektiven Einschätzung der Lage der Partei ,zeigt [die Parteiführung] mögliche Reaktionsstrategien auf und kann zudem externe Organisationserwartungen in innerparteilich pass- bzw. anschlussfähige Reformen übertragen“ (Bukow 2014, S. 136). Methodologisch folgt daraus, dass ,objektive" Gründe als subjektive Begründungen der Parteiführung analysiert werden (Gauja 2017, S. 14-15). Die Rekonstruktion dieser subjektiven Begründungsmuster ist die Grundlage für das Verständnis der Handlungsrationalität der Parteieliten bei der Initiative und Umsetzung der Parteireform (im institutionellen Gefüge der Partei).

Allerdings kann die Parteiführung insbesondere Satzungsänderungen nicht hierarchisch anordnen (Korte und Treibel 2012; Korte et al. 2018). Zur Umsetzung der Reform setzt die Parteiführung zumeist eine (durch den/die Generalsekretär*in geleitete) Kommission ein, die die Deutungen, Begründungen und Zielvorstellungen der Parteiführung in einen Leitantrag überführt, der vom Parteitag beschlossen wird und somit in vorhandene Parteistrukturen eingefügt wird. Dabei besteht immer auch die Chance des Widerstands der mittleren Parteieliten aus den Landes-, Bezirksund Kreisverbänden, sodass Teile einer Reform durchaus noch verändert werden oder scheitern können (Treibel 2013). ${ }^{5}$ Kritik an zentralen Punkten der Reform wird allerdings bereits im Vorfeld des Parteitags so weit wie möglich über informelle Verhandlungen (zum Beispiel innerhalb der nichtöffentlichen Reformkommission) in Kompromisse überführt. So kann sich die Partei während des Parteitags als geschlossene Einheit präsentieren, was als positives Signal von den Medien aufgenommen wird (Treibel 2012, S. 17-18). Bei genereller Einigung über eine Annahme gibt es auf dem Parteitag nur noch Änderungen an Umsetzungsdetails, aber nicht mehr an den Begründungen der Initiative.

Hier zeigt sich deutlich die Prozesshaftigkeit von Parteireformen, welche Auswirkungen auf den Analysezugang hat. Der Beitrag baut daher auf eine interpretative

\footnotetext{
5 So scheiterte Sigmar Gabriel als Parteivorsitzender im Rahmen der Reform 2009-2011 mit seinem Vorhaben, die Partei stärker für Nichtmitglieder zu öffnen (Bukow 2014, S. 145-147).
} 
Form des Process Tracing (Nullmeier 2019). Dabei geht es insbesondere darum, elementare kausale Mechanismen zu identifizieren, die in interpretativer Sichtweise als diejenigen Sinnäußerungen zu verstehen sind, die den Handlungsorientierungen der Akteure unterliegen (Nullmeier 2019, S. 164-165). Diese Mechanismen, verstanden als „Handeln aufgrund von guten Gründen“ (Nullmeier 2019, S. 165), ergeben als Sequenz einen komplexen kausalen Mechanismus, der die Handlungen auf der Mikroebene mit denen auf den darüberliegenden Ebenen verbindet. Forschungspraktisch wird dem Vorgehen von Nullmeier gefolgt, indem zunächst eine Chronologie der Ereignisse dargestellt wird (Abschnitt „Genese der Onlinethemenforen im Reformprozess“), um anschließend die elementaren kausalen Mechanismen, hier verstanden als legitimatorische Begründungsmuster der Parteiführung gegenüber der Gesamtpartei, in Form von Erzählsträngen aufzubereiten (Abschnitt „Begründungen der Einführung von Onlinethemenforen“).

Der Forschungszugang beleuchtet die „Blackbox“ der Handlungen von Parteiakteuren auf der Mesoebene, die zwischen schwer erfassbaren „Gründen“ der Reform (in der Parteiumwelt) und dem hier zu erklärenden Phänomen der Einführung von Onlinethemenforen steht. Bei der Analyse kann plausibel angenommen werden, dass die von der Parteiführung in einer anonymisierten Befragung vorgebrachten Begründungen ihre Einschätzung der Lage der Partei sowie die daraus geschlussfolgerten Handlungsbedarfe wiedergeben, die den Mechanismen des Reformprozesses am nächsten kommen. Noch wesentlich umfassender als öffentlich in Parteidokumenten können sie dabei Kritik, Zweifel, Lernprozesse oder innerparteiliche Auseinandersetzungen reflektieren. Die Begründung der Reform bildet dabei zugleich die Legitimationsgrundlage der Umsetzung über Kommissionen, Leitanträge und Parteitagsbeschlüsse. So wird sich der Analyse eines Mechanismus der Parteireform über die Rekonstruktion von Handlungen der Parteiakteure im Reformprozess so weit wie möglich angenähert. ${ }^{6}$

\subsection{Fallauswahl und Verallgemeinerungspotenzial}

Die SPD ist als Einzelfall im Bereich Parteireformen in doppelter Hinsicht besonders interessant. Zum einen wurden in der vergangenen Reformrunde der SPD 2009-2011 starke innerparteiliche Widerstände gegen die Reform beobachtet, was letztlich zu einer Abschwächung der Reformergebnisse im Vergleich zu den von der Parteiführung angekündigten Maßnahmen führte (Bukow 2014, S. 147-148). Die nur sehr verhalten eingeführten Reformen sind Ausdruck eines der SPD unterstellten Strukturkonservatismus, der zwar bei allen Parteien angenommen wird (Bukow 2013a, S. 13), bei der SPD allerdings zuletzt deutlich sichtbar wurde (Jun 2018). Im Umkehrschluss stellt dies die in diesem Fall beleuchteten Mechanismen, die zu einer erfolgreichen Verankerung von Onlinethemenforen in der Satzung führten, als wirkmächtig dar. Sie können daher als Ansatzpunkt für eine verallgemeinerbare Theorie von Parteireformen gelten, die an anderen Parteien getestet werden kann (Nullmeier 2019, S. 168).

\footnotetext{
${ }^{6}$ Damit schlägt der Beitrag einen Anschluss der Forschung zu Parteireformen an die rekonstruktive und interpretative Politikforschung (Nullmeier 2019; Schwartz-Shea und Yanow 2013) vor.
} 
Zum anderen sind die hier betrachteten Onlinethemenforen als besonders neuartige Entwicklung aufzufassen, da es sich um die Einrichtung und statuarische Verankerung einer vollständig digitalen Parteistruktur handelt - eine „digitale Reform“ (siehe Abschnitt „Analoge und digitale Reformen“). Der digitale Charakter der Reformmaßnahme macht neuartige Begründungs- und Legitimationsmuster der Parteiakteure im Entscheidungsprozess erwartbar, die mit neuartigen Entwicklungen im Rahmen der digitalen Transformation von Politik und Gesellschaft zusammenhängen. ${ }^{7}$ Digitale Kommunikationsinstrumente schüren Hoffnungen auf neue Möglichkeiten demokratischen Austauschs zwischen Parteien und ihren Unterstützer*innen (Jun 2009, S. 203) und könnten tief greifende Veränderungen der Organisationsstrukturen von Parteien nach sich ziehen (Chadwick und Stromer-Galley 2016), die allerdings aus demokratietheoretischer Perspektive auch kritisiert werden können (Gerbaudo 2019; Diehl 2018). In einer Phase, die rückblickend als Formationsphase digitalisierter Parteien gelten könnte, sind Erkenntnisse darüber, warum Parteien Reformen digitaler Art durchführen, daher besonders interessant. Auch sie können als Ansatzpunkt einer Theorie digitaler Reformen fungieren, die an anderen Fällen in anderen Parteien überprüft werden können.

Zur Verallgemeinerung der Erkenntnisse in Richtung einer Theorie der (digitalen) Parteireformen wird wiederum auf den Forschungsansatz des interpretativen Process Tracing verwiesen (Nullmeier 2019, S. 166). Da die in diesem Beitrag identifizierten Sequenzen elementarer kausaler Mechanismen unter spezifischen Akteurs- und Bedingungskonstellationen wirkmächtig wurden, können andere Fälle nach ähnlichen Fallkonstellationen abgesucht werden, um darin festzustellen, ob ähnliche kausale Sequenzen beobachtbar sind. Somit sind die erarbeiteten Erkenntnisse als erster Ansatzpunkt eines solchen Unterfangens zu verstehen, keineswegs aber bereits als vollständige Theorie. Es ist nicht auszuschließen, dass in weiteren Fällen neue Erkenntnisse bezüglich einer spezifischen Gewichtung der Mechanismen oder besonderen Bedingungen ihres Auftretens festgestellt werden. Somit sind die hier erarbeiteten Mechanismen ein erster theoriebildender Schritt, der zugleich auf bestehende Erkenntnisse zu Gründen für Parteireformen (insbesondere Gauja 2017) aufbaut.

\subsection{Datengrundlage und Erhebungsmethode}

Die Begründungen für die Einführung der Onlinethemenforen werden aus den Aussagen der Reforminitiator*innen in der Parteiführung rekonstruiert. Hauptsächliche Datengrundlage sind acht ca. einstündige Expert*inneninterviews aus dem Zeitraum Februar 2019 bis Februar 2020. Als Erhebungsmethode der Befragung wurden teilnarrative Expert*inneninterviews gewählt, da sie einen Mittelweg zwischen größtmöglicher Offenheit und Strukturierungen durch den/die Forscher*in bietet (Helfferich 2014, S. 562-563; Flick 2017; Gläser und Laudel 2010). Sie sind somit gut geeignet für interpretative Forschungsdesigns. Da angenommen wird, dass Expert*innen über „spezialisiertes Sonderwissen“ (Meuser und Nagel 2009, S. 467)

\footnotetext{
${ }^{7}$ Dies begründet auch das offene, induktive Vorgehen gegenüber den Daten (siehe Abschnitt „Auswertungsmethode").
} 
verfügen, wurden für den Beitrag Mitglieder und Mitarbeiter*innen des SPD-Parteivorstands interviewt, die im institutionellen Entscheidungsgefüge der Partei als Initiator*innen der digitalen Reform auftreten.

Ergänzend wurden Parteidokumente in Form von Antrags- und Beschlussbüchern der Parteitage in Berlin 2017 und 2019 herangezogen. Sie dienen in der Partei hauptsächlich der Information über das formale Beschlussverfahren und sind sehr sachlich gehalten und stark formalisiert aufgebaut. In der Analyse dienen sie der Rekonstruktion des Entscheidungsprozesses, da dort nachvollziehbar ist, welche Anträge die einzelnen Gliederungen wie Bundesvorstand, Landes-, Bezirks- oder Unterbezirksverbände sowie Ortsvereine eingebracht haben und wie die Delegierten (inklusive des Vorstands) darüber entschieden haben. Damit dienen sie primär der Kontextualisierung der Analyse.

An einigen wenigen Stellen enthalten die Anträge und Beschlüsse allerdings ebenso wie die Aussagen in den Interviews Begründungen der Einführung von Onlinethemenforen. Verstanden werden diese als „Mittel der Kommunikation“ (Flick 2017, S. 324) der jeweiligen Autor*innen der Dokumente, d.h. des Parteivorstands, des Parteitags oder einzelner Parteigliederungen. Damit gehen sie über den Zweck einer Information hinaus und dienen ebenfalls legitimatorischen Zwecken gegenüber der Gesamtpartei und können in die Inhaltsanalyse der Begründungsmuster einbezogen werden.

\subsection{Sampling}

Das Sampling der Interviewpartner*innen erfolgte als theoretisches Sampling (Flick 2017, S. 158-160; Merkens 2017, S. 295-297). Das theoretische Sampling zielt auf die qualitative Repräsentation der Vielfältigkeit der Perspektiven auf das zu untersuchende Phänomen und zugleich auf die Suche nach gemeinsamen Motiven in den Aussagen der Akteure (Kruse 2015, S. 241-243). Die Auswahl erfolgte somit auf Basis der Wahl eines ersten Falls - eine Person, von der u. a. durch vorherige Forschung bekannt war, dass sie im digitalen Reformprozess involviert ist. Die weiteren Interviewpartner*innen wurden durch Nachfrage bei den jeweils vorherigen Interviewpartner*innen ausgewählt, um weitere beteiligte Personen zu identifizieren, die zugleich die richtigen Personen zur Erhebung von Teilaspekten der Begründungsmuster waren. Das Sampling wurde beendet, als der Eindruck einer ,theoretischen Sättigung" entstand (Flick 2017, S. 161), d.h., als keine neuen Begründungen der digitalen Reform mehr genannt wurden und sich vorherige Begründungen widerspruchsfrei wiederholten.

Alle interviewten Personen waren im Untersuchungszeitraum Mitglieder des Parteivorstands oder Mitarbeiter*innen der Parteizentrale mit Bezug zur Einführung digitaler Technologie oder Bezug zur Organisationsreform. Eine Person war weder Vorstandsmitglied noch Mitarbeiter*in, sondern in beratender Funktion für den Vorstand tätig und hierüber an der Entscheidungsfindung beteiligt. Mehrere interviewte Personen hatten zudem Einblick in die Arbeit der organisationspolitischen Kommission und waren teils selbst dort involviert. 


\subsection{Auswertungsmethode}

Beide Textarten (Interviews und Dokumente) wurden inhaltlich analysiert, indem sie einem doppelten Codierprozess unterzogen wurden. Zunächst wurden per ,descriptive coding“" (Saldaña 2009, S. 70-72) Begründungen für die Einführung der Onlinethemenforen thematisch voneinander abgegrenzt, um einen Überblick über die verschiedenen Begründungen zu erhalten. Dieser Codierschritt ist von großer Offenheit gegenüber den Daten geprägt, um die Möglichkeit zu eröffnen, neuartige, der bestehenden Literatur bislang unbekannte Motive zu entdecken. Anschließend wurde per „process coding“ (Saldaña 2009, S. 77-79) eine Prozessperspektive eingenommen, um die Begründungen der digitalen Reform mit intendierten Wirkungen zusammenzubringen und so mehr darüber zu erfahren, wie die Parteiakteure die neue Parteistruktur mit der aktuellen Lage der Partei und den derzeitigen Strukturen der Willensbildung und Entscheidungsfindung in Verbindung setzen. So kann die Analyse über auslösende, mechanische „Faktoren“ hinausgehen und Begründungen als legitimatorischen Teil von Handlungen verstehen, die an einen Handlungskontext rückgebunden sind. Inhaltlich wurde herausgearbeitet, nach welcher Logik der Akteure beispielsweise die Wahlniederlage von 2017 ein Grund für die Parteireform sein kann.

Der Untersuchungszeitraum ${ }^{8}$ der Studie war Oktober 2017 bis Dezember 2019, da in diesem Zeitraum die Parteireform \#SPDerneuern durchgeführt wurde, in deren Rahmen die Onlinethemenforen eingeführt wurden. Zugleich fallen in diese Zeit die Amtsantritte des neuen Generalsekretärs Lars Klingbeil (Dezember 2017) und der Parteivorsitzenden Andrea Nahles (April 2018), die als maßgebliche Initiator*innen und Unterstützer*innen der Reform gelten. Während Nahles im Juni 2019 ihr Amt aufgab, war Klingbeil während der gesamten Reformperiode im Amt. Überlagert wurde die Reformperiode teilweise vom Mitgliederentscheid über eine neue Vorsitzendendoppelspitze der Partei - die Reformen wurden dennoch parallel unter der Leitung des Generalsekretärs Klingbeil vorangetrieben.

\section{Empirische Analyse}

\subsection{Genese der Onlinethemenforen im Reformprozess}

Als Urheberin der Idee der Onlinethemenforen gilt die Basisinitiative SPD++, die unmittelbar nach der Bundestagswahl 2017 für dieses Vorhaben warb. Im Leitantrag „Die \#SPDerneuern: Unser Weg nach vorn“ für den Bundesparteitag im Dezember 2017 wurden sie bereits vom Parteivorstand als mögliches neues Instrument mit Antragsrecht erwähnt (SPD-Parteivorstand 2017, S. 19). Generalsekretär Klingbeil lud

\footnotetext{
8 Als Untersuchungszeitraum gilt hier der Zeitraum, der aus dem Erkenntnisinteresse des Beitrags abgeleitet wird und über den mit den Interviewpartner*innen gesprochen wurde. Er ist zu unterscheiden vom Interviewzeitraum, der sich nur teilweise mit dem Untersuchungszeitraum deckt (siehe Abschnitt „Datengrundlage und Erhebungsmethode“). Die Interviews wurden somit teils prozessbegleitend, teils rückblickend geführt.
} 
im Frühjahr 2018 Vertreter*innen der Initiative zu Konsultationen über Onlinethemenforen ein ${ }^{9}$ und diskutierte Ideen der Umsetzung eines solchen Instruments. Nach dem offiziellen Start der Parteireform im April 2018 wurde die von Klingbeil geleitete organisationspolitische Kommission (OrgaKomm) ${ }^{10}$ das zentrale Instrument zur Ausarbeitung der Reformen, deren Vorschläge zur Organisationsreform in einen Leitantrag des Vorstands für den Bundesparteitag 2019 einflossen.

Zeitgleich wurden im Laufe des Jahres 2018 vier sogenannte Lenkungsgruppen eingerichtet, die programmatische Reformen erarbeiten sollten. In Bezug auf die anstehenden Organisationsreformen war dies insofern bemerkenswert, als dass damit im September 2018 die Einrichtung des sogenannten Debattenportals ${ }^{11}$ einherging, ein parteiinternes Onlineforum, in dem die Parteimitglieder die programmatische Reform der SPD umgehend „,digital mitdiskutieren“ konnten, so Generalsekretär Klingbeil (Vorwärts 2018). Dies diente den angedachten Organisationsreformen als Vorabexperiment, das Erkenntnisse brachte, wie digitale Kommunikation und Beteiligung in der SPD gestaltet werden könnten. Kritisiert wurde allerdings von Teilnehmer*innen, dass die Übernahme der Inhalte durch die Parteiführung wie in früheren digitalen Experimenten (Hanel und Marschall 2014) völlig intransparent geschah.

Das stark kritisierte Experiment „Debattenportal“ galt als warnendes Beispiel bei der Entscheidung über die Anbindung der Onlinethemenforen an die bestehenden Parteistrukturen in der OrgaKomm. So wurde seit dem Frühjahr 2019 wieder vermehrt von der Einrichtung sogenannter Onlinethemenforen gesprochen, die bereits in den Parteitagsbeschlüssen von 2017 Erwähnung fanden (zu Vorformen: Bieber 2014; Hanel und Marschall 2014). ${ }^{12}$ Es wurde betont, dass es die Frage der institutionellen Anbindung sei, die darüber entscheiden könnte, ob die Onlinethemenforen unter den Mitgliedern als effektives Beteiligungsinstrument wahrgenommen und akzeptiert würden. Vertreter*innen der Basisinitiative SPD++ forderten daher ein Antragsrecht für Bundesparteitage sowie das Recht, stimmberechtigte Delegierte als Repräsentanten der Mitglieder in den Onlinethemenforen zum Parteitag entsenden zu können.

Das Ergebnis der organisationspolitischen Kommission war ein Kompromiss: Zwar sollten die Onlinethemenforen Antragsrecht und Delegierte bekommen. Die Delegierten sollten allerdings nur als beratende Delegierte am Parteitag teilnehmen, d. h. zwar redeberechtigt, aber nicht stimmberechtigt sein. Damit wurden sie letztlich

\footnotetext{
9 Vgl. Blogpost von Henning Tillmann, Vorsitzender SPD++: https://spdplusplus.de/spd-debattenportal. Zugegriffen: 27.03.2020.

10 Die organisationspolitische Kommission bestand aus verschiedenen Verterter*innen der Gesamtorganisation: Neben dem Parteivorstand und Vertreter*innen der Bezirke und Landesverbände gehören Vertreter*innen von Arbeitsgemeinschaften sowie kommunalpolitische und digitalpolitische Expert*innen zu diesem Gremium.

11 Eine Analyse zu Entstehung, Ausgestaltung und Nutzung des Debattenportals liefern de Nève und Ferch (2019, S. 58-60).

12 Als im September 2018 das Debattenportal vorgestellt wurde, bestand unter den Mitgliedern Unklarheit, ob es als Umsetzung der 2017 angekündigten Onlinethemenforen zu verstehen sei (siehe SPD++ 2018: „Debattenportal: Die SPD hat nun online-basierte Themenforen! ... oder doch nicht?“, online unter: https:// spdplusplus.de/spd-debattenportal. Zugegriffen: 13.12.2019). Nach der negativen Erfahrung mit dem Debattenportal wurde dieses dann aber als Vorläufer von veränderten Onlinethemenforen dargestellt.
} 
auf eine Stufe mit Arbeitsgemeinschaften wie den Jusos oder der Arbeitsgemeinschaft Sozialdemokratischer Frauen (ASF) gestellt, die ebenfalls über Antragsrecht und beratende Delegierte mit Rederecht verfügen. ${ }^{13}$ Gruppen von Mitgliedern im Internet erhielten so eine Möglichkeit der Mitsprache, die gehört werden kann allerdings ohne Möglichkeit, über selbst erarbeitete programmatische Positionen abzustimmen (SPD-Bundesparteitag 2019). Bemerkenswert an der Organisationsreform ist, dass erstmals digital organisierte Gruppen von Parteimitgliedern über die Onlinethemenforen ein Antragsrecht auf Bundesparteitagen erhalten ( $\S 10$, Abs. 3 Organisationsstatut SPD), das bislang ausschließlich ortsgebundenen Gliederungen ${ }^{14}$ vorbehalten war. Am 06.12.2019 wurde die Einführung der Onlinethemenforen im Rahmen einer größeren organisationpolitischen Reform auf dem Bundesparteitag in Berlin beschlossen (SPD-Bundesparteitag 2019).

\subsection{Begründungen der Einführung von Onlinethemenforen}

In den untersuchten Interviews und Parteidokumenten zeigten sich insgesamt sechs verschiedene Begründungsmuster, auf die nun näher eingegangen wird:

1. Wahlniederlage 2017 ,

2. Erwartungshaltung der eigenen Mitglieder an veränderte Partizipationsnormen,

3. Beitritt neuer Mitglieder,

4. themenorientierte, digitale Vernetzung der Mitglieder,

5. ein „Ort der Wahrheit“ neben Massenmedien und sozialen Medien,

6. Effizienz der Parteiarbeit.

\subsubsection{Wahlniederlage 2017}

Mehrfach wird als allgemeiner Reformgrund, aber auch spezifisch zur Einführung digitaler Instrumente die Niederlage bei der Bundestagswahl 2017 genannt. Allerdings habe es bereits davor Überlegungen in Richtung einer umfassenden Organisationsreform gegeben:

Die herbe Wahlniederlage 2017 war der letzte Auslöser würd' ich fast sagen. Also, [...] gewisse Analysen, was die Partei oder die SPD [...] besser machen könnte, eigentlich, liegen auch seit den letzten Wahlniederlagen auf dem Tisch. Also, seit 2009, 2013, dann 2017. So man hat nach den Parteitagen immer [...] aufgeschrieben und abgestimmt: Man möchte irgendwie jünger, weiblicher, digitaler andere Gruppen binden an die Partei. [...] Also eigentlich hat man kein

\footnotetext{
13 Im Statut werden sie im gleichen Abschnitt wie die Arbeitsgemeinschaften aufgeführt (§ 10 SPD-Parteistatut).

14 Auch Jusos und ASF sind trotz ihres ,thematischen“ Fokus in diesem Sinne als ,ortsgebunden“ zu bezeichnen, da sie eine dem Föderalismus nachempfundene Hierarchie aufweisen, die die Arbeitsgemeinschaften nach regionaler Logik in Landesverbände/Bezirksverbände, Unterbezirke und Ortsgruppen gliedert, welche jeweils Vorstände und Delegierte für die nächsthöheren Ebenen wählen.
} 
Analysedefizit gehabt, sondern eher 'n Ernsthaftigkeitsdefizit bei der Umsetzung dann letztendlich (Interview \#21). ${ }^{15}$

Betont wird insbesondere die Wahrnehmung in der Parteiführung, dass man es in der langjährigen Regierungszeit vor 2017 nicht geschafft habe, sich inhaltlich und organisatorisch weiterzuentwickeln und sich so an gesellschaftliche Entwicklungen - auch den Wunsch nach digitaler Beteiligung - anzupassen, was zu immer gravierenderen Wahlniederlagen geführt habe (Interview \#42). Die Schlussfolgerung aus der Wahlniederlage 2017 sei neben der Anbahnung einer großen Parteireform zunächst auch die Ablehnung eines erneuten Eintritts in die Große Koalition (,GroKo“) gewesen (Interview \#42). Als der Druck zum Eintritt in die Koalition nach dem Scheitern der Jamaika-Verhandlungen stieg, wurde innerhalb der Parteiführung ein Kompromiss zwischen Befürworter*innen und Gegner*innen der Koalition erarbeitet. Dieser bestand darin, dass GroKo-Befürworter*innen der zuvor teils skeptisch betrachteten Ausweitung digitaler Beteiligungsmöglichkeiten für Parteimitglieder im Rahmen einer Parteireform zustimmten:

Also so 'n bisschen, böse gesagt, Tauschhandel, [...] sozusagen: Ich geb euch mehr digitale Beteiligung innerhalb der Partei, aber dafür dürfen wir in die GroKo gehen (Interview \#21).

Bereits im Herbst 2018 wurde die „Frage der Ernsthaftigkeit“" (Interview \#21) bei der Umsetzung digitaler Instrumente virulent, da zunächst nur eine intransparente und willkürliche Übernahme von Inhalten des Debattenportals durch die Parteiführung vorgesehen war (Interview \#21, \#60, \#33). Diese Haltung führte zum Scheitern des Debattenportals, da es ähnlich wie frühere digitale Beteiligungsinstrumente (Hanel und Marschall 2014) als unverbindliches Feedbackinstrument zum Stimmungstest für Vorhaben des Vorstands begriffen wurde und nicht an den institutionell verankerten Entscheidungsprozess der Partei angebunden wurde (Interview \#60, \#33).

Dennoch blieb der Konsens über die Einführung digitaler Beteiligungsinstrumente erhalten. Dadurch konnte eine Weiterentwicklung des Debattenportals zu Onlinethemenforen vorangetrieben werden. Dies galt insbesondere deswegen, weil die Reformbefürworter ihre Ideen in den Kontext einer umfassenden Organisationsreform zur Verbesserung künftiger Chancen auf Wahlerfolge stellten. Denn digitale Beteiligungsmöglichkeiten allein wurden intern keineswegs als Garant für künftige Wahlerfolge interpretiert:

Also ich glaube nicht, [...] dass die SPD wieder auf über $30 \%$ kommt, nur weil sie Online-Tools macht. Weil, das interessiert draußen auch keinen. [...] Das heißt nicht automatisch, dass uns das irgendwie 3\% mehr bringt bei den Bundestagswahlen oder bei irgendwelchen Wahlen (Interview \#21).

Vielmehr war die Einführung des Debattenportals als Vorläufer der Onlinethemenforen indirekte Folge der Wahlniederlage 2017: Nicht die digitale Technologie

15 Die Interviews wurden aus Anonymisierungsgründen mit einer Zufallszahl zwischen 1 und 99 versehen. Es wurden nicht die Zahlen 1 bis 8 verwendet, um die Suggestion einer Reihenfolge zu vermeiden. 
selbst galt als Garant für künftige Wahlerfolge, sondern als geeignetes Mittel zum Zweck der inhaltlichen Neuaufstellung unter Beteiligung möglichst großer Teile der Mitglieder, ,damit das nicht nur ein kleiner Expertenkreis immer ausarbeitet“ (Interview \#55). Daher zielte die Einführung digitaler Instrumente darauf ab, inaktive Parteimitglieder zum Engagement in der Partei zu befähigen und gleichzeitig auf Erwartungen an digitale Beteiligungsmöglichkeiten innerhalb und außerhalb der Partei zu reagieren.

\subsubsection{Erwartungshaltung der Mitglieder an veränderte Partizipationsnormen}

Laut der Parteiführung hätten Bürger*innen eine Erwartung an punktuelle und themenbezogene politische Beteiligung entwickelt, welche den klassischen Partizipationsformen in der Partei teilweise entgegenstünde (Interview \#14, \#79). Daher müsse man auf diese Entwicklung reagieren:

Die ganzen Beteiligungsmöglichkeiten haben sich ja im Prinzip verändert durch das Internet [...]. Und diese Ansprüche sind eben da aus der Gesellschaft, so. Und da muss man dann eben auch nen Ort bieten, als Partei (Interview \#21).

Viele Menschen würden sich zwar gerne in eine Partei einbringen, scheuten aber das langfristige und an klassische Parteistrukturen gekoppelte Engagement: „Und das sind dann Leute, [...] die wollen gar nicht Delegierte werden, die wollen gar nicht auf Kreisparteitage, die wollen gar nicht sich zwei Jahre lang an irgendein Amt binden, aber die sagen: Ich habe hier ein Potenzial und das will ich gerade in meine Partei einbringen“ (Interview \#14). Digitale Beteiligungsformen sind zwar kein Hauptargument für Neueintritte, ihr Fehlen könnte aber die Popularität der Partei verringern (Interview \#21).

Die Dringlichkeit, mit der die Partei nach Veränderungen strebt, wird insbesondere vor dem Hintergrund klar, dass bereits jetzt mangelnde Responsivität herrsche: „Wir haben ja ganz offenbar Fäden in diese Gesellschaft verloren“ (Interview \#60). Somit gestaltet sich die Einrichtung digitaler Instrumente als Suche nach verloren gegangenen und neuen Potenzialen, die in den letzten Jahren unzureichend ausfiel:

Der Zustand der SPD, im Sinne der Partizipation, ist nicht in Ordnung. [...] Wir wollen die Partei offener, jünger, weiblicher und eben auch digitaler machen (Interview \#33).

Dieser Veränderungsdruck wird allerdings nicht nur als externer Druck der Gesellschaft wahrgenommen, sondern auch als organisationsinterner Druck der Mitglieder (Interview \#60, \#42). So wird auch unter den Mitgliedern ein Wandel von Lebenswelten diagnostiziert, der mit der Notwendigkeit räumlicher und zeitlicher Flexibilität einhergehe. Als Beispiele werden junge Eltern genannt, die beruflich stark eingebunden sind, oder auch Studierende im Ausland (Interview \#14, \#21). Die räumlich und zeitlich fixierten Treffen der Ortsvereine seien für die Mitglieder aber bislang der einzige Ort, sich an der Parteiarbeit zu beteiligen, was der neuen Lebenswelt der Parteimitglieder immer weniger entspräche: 
Weil sie einfach keine Lust haben auf, jeden zweiten Montagabend im Goldenen Hirschen um 19:30 Uhr, so. Ist für viele junge Leute, Familien mit Kind und Kegel, nicht so attraktiv vielleicht, da teilzunehmen (Interview \#21).

Hinzu kommt, dass gerade jüngere Mitglieder generell weniger Verständnis für das in den Parteien vorherrschende Territorialprinzip aufbringen. Im Gegenteil nehmen sie eine eher ambivalente Haltung gegenüber dem Ortsverein als genuin erster Anlaufstelle ein. Dieses Aufeinanderprallen des Orts- bzw. Territorialprinzips mit digitalen Beteiligungs- und Vernetzungsformaten bricht mit der klassischen Organisationslogik. „Also der klassische Ort ist der Ortsverein, vor Ort, die regionalen Strukturen. Und da geht man dann eben hin oder auch nicht“" (Interview \#21). Diese Ambivalenz gegenüber dem Ortsverein und klassischen Parteistrukturen wird auch deshalb problematisch gesehen, weil neu eingetretene Mitglieder aus ihren außerparteilichen Zusammenhängen (z. B. Arbeit, soziales Engagement oder Familienleben) andere Formen der Kommunikation und Abstimmung gewohnt sind und diese Verhaltensweisen und Tools in der Partei nicht vorfinden und vermissen.

\subsubsection{Beitritt neuer Mitglieder}

Deutlich wurde die spezifische Erwartungshaltung neuer Mitglieder insbesondere, als mit den guten Umfragewerten nach der Übernahme des Parteivorsitzes durch Martin Schulz (sog. Schulz-Hype) zahlreiche neue, vor allem junge Mitglieder in die SPD eintraten: „Weil es sind viele in die SPD eingetreten, und viele die halt quasi gesagt haben: So jetzt bin ich in der SPD drin, was kann ich jetzt eigentlich tun?“ (Interview \#33). Der entscheidende Schritt, um diese Neumitglieder in der Partei zu halten, sei, dass die Partei der Lebenswelt dieser Menschen entsprechend auf sie zugehe:

Also, reicht es, wenn ein neues Mitglied eintritt in den Ortsverein, oder in der regionalen Struktur, dieses neue Mitglied einfach auf den Mailverteiler zu setzen? Meine Behauptung: Nein (Interview \#21).

Es gehe daher darum, neue Mitglieder unmittelbar anzusprechen und ihnen Möglichkeiten zu eröffnen, in der Partei mitzuarbeiten. Will man einen Generationswechsel mit neuen, insbesondere jungen Mitgliedern schaffen, so sei eine Ausrichtung an deren Beteiligungswünschen unabdingbar. Die unmittelbare Ansprache und Aufforderung zur Mitarbeit habe früher erst im Ortsverein stattgefunden, soll in Bezug auf neue, junge Mitglieder nun allerdings noch früher auf digitalem Weg passieren (Interview \#21).

Ein Wunsch der Parteiführung ist daher, dass nicht nur die Parteimitglieder erreicht werden, die sich innerhalb dieser klassischen Strukturen bewegen und deren Lebenswelt damit vereinbar ist, sondern die Forderung nach Beteiligungsmöglichkeiten der weiteren Parteimitglieder ernst zu nehmen und die Struktur der Partei entsprechend anzupassen. Es wird bemängelt, dass nur ein geringer Teil der Mitglieder überhaupt erreicht wird und man hier Potenzial verschenke (Interview \#14). 


\subsubsection{Themenorientierte, digitale Vernetzung der Mitglieder}

Es geht der Parteiführung allerdings nicht ausschließlich um Flexibilisierung der Partizipationsmöglichkeiten für die Mitglieder im Sinne zeitlicher und räumlicher Freiheiten über digitale Instrumente. Ein weiteres Motiv ist die Ermöglichung themenorientierter Vernetzung mit Gleichgesinnten (Interview \#60, \#87). Denn viele Menschen, so die Parteiführung, wollen sich nicht mit den Themen auseinandersetzen, die ihnen zufällig begegnen, weil sie in ihrem Ortsverein aufgrund der wohnortabhängigen (und damit zufälligen) Mitgliederzusammensetzung dominieren. Ein Motiv politischen Engagements der Parteimitglieder sei zunehmend eine Expertise in einem spezifischen thematischen Bereich, erworben im beruflichen oder privaten Kontext, die in politisches Engagement überführt werden soll (Interview \#14, \#33). Hierfür könnten digitale Instrumente Anknüpfungspunkte bieten, die der Ortsverein nicht leisten kann: „Dass eben sozusagen entlang von Themenschwerpunkten ne überregionale Vernetzung möglich ist“" (Interview \#60).

Dieser Gedanke spielte konkret bei der gewünschten programmatischen Neuaufstellung nach der Wahlniederlage 2017 eine Rolle. Denn statt einer Beteiligung der Mitglieder an der Erarbeitung neuer Positionen über digitale Instrumente hätten auch schlicht Anträge der Ortsvereine oder Kreis- und Landesverbände oder beispielsweise die Einberufung von Regionalkonferenzen als bereits bekannte Möglichkeiten in Betracht gezogen werden können. Es wurde allerdings dezidiert auf digitale Technologie gesetzt, um einen deutschlandweiten Zusammenschluss der Mitglieder sowie eine Themenorientierung zu erreichen (Interview \#55). Regionalkonferenzen wurden zudem abgelehnt, da sie in der Vergangenheit in Situationen der Krise und der Führungsschwäche eingesetzt wurden - ein Bild, was die Parteiführung in einer proklamierten Situation des Aufbruchs vermeiden wollte (Interview \#55). Als Begründung für die Einführung des Debattenportals und dessen Weiterentwicklung zu den Onlinethemenforen wurden somit (neben ihrer vermuteten positiven Konnotation in großen Teilen der Mitglieder) explizit die Vorteile der ihnen unterliegenden digitalen Technologie genannt, welche einen neuen Zusammenschluss von Parteimitgliedern ermöglicht, der in der bisherigen, analogen Parteistruktur nicht möglich war.

\subsubsection{Ein „Ort der Wahrheit“ neben Massenmedien und sozialen Medien}

Eine wichtige Rolle spielt die Wahrnehmung der Parteiführung, dass themenbezogene Diskussionen, auch der eigenen Parteimitglieder, sich mangels parteiinterner Alternativen in die kommerziellen sozialen Netzwerke Facebook und Twitter verlagern. Das wird höchst kritisch gesehen:

Ist aber schon ' $\mathrm{n}$ Thema, worüber man intensiv nachdenken muss: Wo ist eigentlich tatsächlich der Austauschort? Es findet ja auf Facebook einiges statt, es findet auf Twitter einiges statt an Austausch und Vernetzung, aber es ist nicht zielgerichtet und es ist nicht nützlich (Interview \#60).

Ziel sei daher, den eigenen Parteimitgliedern einen digitalen, themenorientierten Austausch zu ermöglichen, der produktiv als Konsensfindung zwischen politischen 
Positionen innerhalb der Partei verlaufe und erst im Anschluss in Diskussion mit der Öffentlichkeit trete: „Brauchen wir vielleicht doch einen Ort der Wahrheit, wo man untereinander die Sachen klären kann. Und bekommt man dann vielleicht auch wieder das hin, das auf den Kanälen, wo eben andere auch zuschauen, [...] das Werben für unsere Themen gelingt“" (Interview \#60). Hier spielt auch das digitale Spezifikum eine Rolle, dass der Austausch nicht erst an bestimmten Terminen stattfindet (wie z. B. auf einem Parteitag), sondern über Onlinethemenforen permanent stattfinden kann, wie in den sozialen Medien üblich und von den Mitgliedern gewünscht (Interview \#42).

Die Onlinethemenforen sind so als Puzzleteil einer größeren Strategie der Außenkommunikation zu verstehen. Im Kern geht es darum, dass Parteimitglieder bislang unter den Augen der Öffentlichkeit über inhaltliche Positionen streiten und dabei starke Unzufriedenheit über den Kurs der Partei äußern, was von Medien im Sinne ihrer Aufmerksamkeitslogik als innerparteiliche Zerrissenheit und eine Geschichte des Niedergangs dargestellt wird. Dieses Bild wiederum prägt auch die eigenen Parteimitglieder, die eine negative Grundhaltung gegenüber der eigenen Partei entwickeln, so die Annahme der Parteiführung. Es besteht innerhalb der Parteiführung eine große Unzufriedenheit über die in den Medien verbreitete Geschichte des Niedergangs der SPD, die sich hartnäckig hielte (Interview \#14, \#60).

Als Lösung dieses wahrgenommenen Problems zielt der Vorstand darauf ab, Botschaften direkt und ungefiltert an die eigenen Mitglieder zu senden, wodurch eine Deutungshoheit über Themen und das Image der eigenen Partei angestrebt wird. So wird allgemein davon gesprochen, digitale Instrumente würden dem Parteivorstand dabei helfen, ,,in der Partei zu kommunizieren, Botschaften zu verbreiten, über Prozesse zu informieren“ (Interview \#14), wofür beispielsweise ein Telegram-Kanal ${ }^{16}$ eingerichtet wurde, Mailinglisten und die sozialen Medien genutzt werden (Interview \#60). Dabei ist dem Parteivorstand durchaus bewusst, dass die Willensbildung an den digitalen „Orten der Wahrheit“, wie künftig in den Onlinethemenforen, durch ihre parallele Aussendung von Botschaften auf anderen digitalen Kanälen beeinflusst wird (Interview \#87). Dies wird im Sinne einer kohärenteren digitalen Willensbildung und einer Reduzierung von sichtbarem Streit in öffentlichen digitalen Medien allerdings in Kauf genommen. Eine Verlagerung des inhaltlichen Austauschs in die Onlinethemenforen wäre ein Schritt in diese Richtung.

\subsubsection{Effizienz der Parteiarbeit}

Weiterhin wird die Einführung digitaler Instrumente von der Parteiführung als neue Möglichkeit betrachtet, die gesamte Parteiorganisation effizienter zu machen. Vor dem Hintergrund gestiegenen Kostendrucks in der Partei, was Schatzmeister Dietmar Nietan auf dem Parteitag 2019 als zentralen Grund für Organisationsreformen benannte, erlangt das Begründungsmotiv Digitalisierung aus Effizienzgründen große Bedeutung. In Bezug auf die Arbeit der hauptamtlichen Mitarbeiter*innen per

\footnotetext{
16 Der WhatsApp-Eigner Facebook untersagte über eine Änderung der Nutzungsbedingungen das Anbieten von Parteikanälen auf WhatsApp ab Dezember 2019. Daraufhin verlagerte die SPD-Parteizentrale den zuvor über WhatsApp angebotenen Informationsdienst zum Messenger Telegram.
} 
Videokonferenzen oder kollaborativen Textbearbeitungstool werden beispielsweise Geschwindigkeitsvorteile betont. Obwohl sich dies derzeit auf die Arbeit in den Parteizentralen beschränkt, besteht der Wunsch, diese Art des gemeinsamen digitalen Arbeitens auf die gesamte Partei auszudehnen (Interview \#14, \#60).

So beschäftigte sich eine Arbeitsgruppe der organisationspolitischen Kommission, die die Reformvorschläge für den Parteitag 2019 vorbereitete, explizit mit der Effizienzsteigerung durch digitale Instrumente: „Wo können wir denn [...] sowohl Organisationsgewinne als auch ökonomische Gewinne erzielen“ (Interview \#60). Zwar gebe es bereits einige Möglichkeiten ganz selbstverständlich bereits durch die Nutzung von E-Mails (Interview \#14) und auch durch die zentrale Mitgliederverwaltung (Interview \#60), dennoch bestehe beispielsweise beim Thema digitales Antragswesen ein großer Reformbedarf, der in der Vorbereitung von Parteitagen große Effizienzgewinne verspräche. Im Sinne einer angestrebten Digitalisierung des Antragswesens sind die Onlinethemenforen ein erster Ansatz, programmatische Antragsarbeit ins Internet zu verlagern. Denn dort sind sie effizienter und kostengünstiger umzusetzen als während zeitlich und örtlich fixierter Ortsvereins- oder Arbeitsgruppentreffen. Die Onlinethemenforen sind somit als Beginn einer langfristig geplanten Einführung eines digitalen Antragswesens zu verstehen, das auf Zeit- und Materialersparnisse abzielt und die Parteiorganisation somit effizienter machen soll.

\subsection{Gründe für die digitale Organisationsreform 2017-2019}

Inwiefern schließen die Begründungsmuster an die drei von Gauja (2017) benannten Ebenen an (Tab. 2, siehe Abschnitt „Warum reformieren sich Parteien?“)? Die empirische Analyse zeigte zunächst, dass der von Gauja (2017, S. 50-75) als Reformtreiber genannte Bereich Parteienwettbewerb und Wahlen durch die Ergebnisse der Fallstudie ausdifferenziert wird. Zwar galt die Wahlniederlage 2017 als Auslöser

Tab. 2 Differenzierung und Erweiterung von Gründen für Parteireformen. (Eigene Darstellung)

\begin{tabular}{|c|c|c|}
\hline Begründungsmuster & $\begin{array}{l}\text { Anschluss an „,Gründe“" } \\
\text { (Gauja 2017) }\end{array}$ & Differenziert oder erweitert Gründe um ... \\
\hline Wahlniederlage 2017 & $\begin{array}{l}\text { Parteienwettbewerb und } \\
\text { Wahlen }\end{array}$ & - \\
\hline „Ort der Wahrheit““ & $\begin{array}{l}\text { Parteienwettbewerb und } \\
\text { Wahlen/innerparteiliche } \\
\text { Gründe }\end{array}$ & $\begin{array}{l}\text { Digitale Kommunikation in sozialen Medien, } \\
\text { Kontrolle über innerparteiliche Kommunika- } \\
\text { tion }\end{array}$ \\
\hline $\begin{array}{l}\text { Erwartung eigener Mit- } \\
\text { glieder an „Modernität“ } \\
\text { der Beteiligung }\end{array}$ & $\begin{array}{l}\text { Gesellschaftliche Er- } \\
\text { wartungen }\end{array}$ & $\begin{array}{l}\text { Nicht nur mehr, sondern auch ,,andere“ Be- } \\
\text { teiligung }\end{array}$ \\
\hline $\begin{array}{l}\text { Angebot für neu eingetre- } \\
\text { tene Mitglieder }\end{array}$ & $\begin{array}{l}\text { Gesellschaftliche } \\
\text { Erwartungen/ } \\
\text { innerparteiliche Gründe }\end{array}$ & $\begin{array}{l}\text { Neue (auch digitale) Vorstellung von Par- } \\
\text { teiorganisation, erhöhte Responsivität der } \\
\text { Partei }\end{array}$ \\
\hline $\begin{array}{l}\text { Themenorientierte Vernet- } \\
\text { zung }\end{array}$ & $\begin{array}{l}\text { Gesellschaftliche } \\
\text { Erwartungen/ } \\
\text { innerparteiliche Gründe }\end{array}$ & $\begin{array}{l}\text { Neue Kommunikations- und Kollaborations- } \\
\text { logik durch digitale Affordanzen - Erweite- } \\
\text { rung der Parteiorganisation }\end{array}$ \\
\hline Effizienz der Parteiarbeit & Innerparteiliche Gründe & $\begin{array}{l}\text { Langfristige Kosten senken durch digitale } \\
\text { Antragsarbeit }\end{array}$ \\
\hline
\end{tabular}


der Reform, allerdings spielte der spezifische Wunsch nach einer stärkeren Kontrolle des innerparteilichen Kommunikationsflusses mit Blick auf zukünftige Wahlen ebenfalls eine bedeutsame Rolle. Insbesondere veränderte Kommunikationsumgebungen in einer von sozialen Medien geprägten digitalen Öffentlichkeit (Dohle et al. 2014; Tenscher und Borucki 2015) beeinflussen die Außendarstellung einer Partei stark, was die Parteiführung zu Reformen führt, die Möglichkeiten der Steuerung digitaler Kommunikationsflüsse ausloten.

Prinzipiell soll die Schlagfertigkeit der Partei im Wahlkampf durch eine möglichst einheitliche Außendarstellung der Partei auch im digitalen Zeitalter gelingen. Ein stärker nach innen, zum „Ort der Wahrheit“, gelenkter digitaler Kommunikationsfluss soll digital sichtbar gewordene Auseinandersetzungen zwischen Parteimitgliedern kanalisieren und vor den Augen der Öffentlichkeit verbergen. Die Kontrolle der Außendarstellung ist Teil eines Professionalisierungstrends, der auch in früheren SPD-Parteireformen, z. B. über stärker zentralisierte interne Kommunikation im Willy-Brandt-Haus, sichtbar wurde (Bukow 2013a, 2013b; Jun 2009).

Insofern ist die jüngste Einführung digitaler Kommunikationsinstrumente als Fortsetzung eines Trends zu deuten: Das Ziel der Erlangung von Deutungshoheit gegenüber den Medien und den eigenen Mitgliedern impliziert auch eine Prägung des Diskurses über die Partei und tagespolitische Ereignisse mit eigenen Botschaften und Erzählungen. Die Parteiführung befindet sich derzeit in einem Lernprozess, wie dieser Streit mithilfe digitaler Instrumente intern produktiv in Richtung eines deliberativen Konsenses gewendet werden kann, sodass die Parteimitglieder in der Öffentlichkeit stärker gemeinschaftlich auftreten. Ob der Ansatz einer intensiv gesteuerten Kommunikation von oben nach unten vor diesem Hintergrund Erfolg versprechend ist, ist unklar. Noch gibt es keine Patentlösungen für eine Ordnung der anarchischen digitalen Kommunikation.

In Bezug auf den von Gauja (2017, S. 76-98) benannten Bereich der veränderten gesellschaftichen Erwartungen an Parteien zeigte sich im untersuchten Fall ebenfalls eine digitalspezifische Ausdifferenzierung von Reformgründen. So nimmt die Parteiführung nicht nur Forderungen nach „mehr“ Beteiligung, sondern auch nach ,anderer" Beteiligung wahr, die mit der digitalen Lebenswelt der Bürger*innen und Mitglieder zu tun hat. Die digitale Reform wurde von der SPD-Parteiführung als Reaktion auf diese Erwartung dargestellt, da sie das Versprechen eines kurzfristigen, themenorientierten Engagements besser einlöst.

Allerdings geht es dabei darum, dass die Organisationsform Ortsverein, aber auch die Arbeitsgemeinschaften den Ansprüchen von digital sozialisierten Neumitgliedern, insbesondere von jüngeren Neumitgliedern, nicht mehr entspricht. Besonders bedeutsam ist die Zeitspanne unmittelbar nach Parteieintritt, in der den Mitgliedern ein zeitgemäßes Beteiligungsangebot gemacht werden muss, um die Chance auf eine Verstetigung der Mitgliedschaft zu wahren. Zugleich muss die Parteiorganisation in diesem Moment ein Beteiligungsangebot bereithalten, das den Erwartungen nach ,,anderer“ als bislang üblicher Beteiligung entspricht. Hier geht es darum, die Affordanzen der digitalen Technologie, die neuen Kommunikations- und Kollaborationslogiken entsprechen, mit der Organisationsform der Partei zu verbinden. Erstmals wurde dabei Abstand vom Territorialitätsprinzip der Partei genommen, welches bislang sowohl die reguläre Parteihierarchie als auch den Organisationsaufbau 
der Arbeitsgemeinschaften prägte. Onlinethemenforen sind stattdessen horizontal vernetzt, durch Asynchronität zeitlich flexibel und kennen keine Vorstands- und Hierarchieebenen auf dem Weg zum Antrag auf dem Bundesparteitag.

Der themenorientierte Zusammenschluss bietet darüber hinaus die erwartete Alternative zu den früheren Repräsentationslogiken der Arbeitnehmer*innen, der Frauen oder der jungen Mitglieder in Arbeitsgemeinschaften, die Themenorientierung nicht spezifisch in Bezug auf Policy-Felder umsetzen. Diese themenorientierte Vernetzung von Mitgliedern als Parallelstruktur zur ortsbezogenen Vernetzung war bereits Thema früherer Reformen, nur nicht digital gedacht. Diskussionen um eine Öffnung für Nichtmitglieder im Jahr 2009 wurden mit der Idee eines Aufbaus themenorientierter Netzwerke begründet: Die Bestrebungen zielten „darauf ab, neue, weniger dauerhafte, sondern punktuelle, themenbezogene Partizipationsformen in politischen Parteien zu eröffnen und damit die nach geographischen Ordnungsprinzipien orientierte Parteistruktur [...] durch den Auf- und Ausbau thematischer Netzwerkstrukturen zu ergänzen“ (Jun 2009: S. 198). Dies konnte durch die Onlinethemenforen letztlich realisiert werden, basierend auf einer Idee aus einer Zeit, als soziale Medien noch in den Kinderschuhen steckten.

In dem von Gauja (2017, S. 28-49) benannten Bereich innerparteilicher Gründe für Reformen konnte, entgegen theoretischer Erwartungen, die These machttaktischer Gründe für Organisationsreformen nicht gestützt werden. Stattdessen ging es im innerorganisatorischen Bereich, neben den genannten Erwartungshaltungen der Neumitglieder, um die Steigerung der Effizienz von Parteiarbeit. Insbesondere, da langfristiger Mitgliederschwund in Kombination mit sinkenden Wahlergebnissen zu finanziellen Notlagen führte, gelten digitale Instrumente als Möglichkeit der ökonomischen Effizienzsteigerung. Digitalisierung aus Effizienzgründen erhält dann große Bedeutung, wenn es darum geht, Entscheidungen schneller, kostengünstiger und unbürokratischer herbeizuführen, etwa durch ein digitalisiertes Antragswesen vor Parteitagen. Zu derlei Vorbereitungen sind die untersuchten Onlinethemenforen ein Ansatz. Dieses Begründungsmuster passt zum allgemeinen Professionalisierungstrend der Parteien, scheint als genuiner Grund für eine Parteireform in den Theorien der Parteireformen bislang allerdings unbeachtet.

\section{Fazit}

\subsection{Auswirkungen der digitalen Reform auf die Organisation der SPD}

Insgesamt ist in Bezug auf die potenzielle Wirkung der Reform zu konstatieren, dass die Onlinethemenforen als eine Ausweitung der Basisbeteiligung interpretiert werden können. Durch die Einrichtung der digitalen Foren im Rang einer Arbeitsgemeinschaft mit Antragsrecht auf Bundesparteitagen und beratenden Delegierten ohne Stimmrecht ist das durchaus der Fall. Allerdings verändert sich der formale Prozess der kollektiven Entscheidungsfindung in der SPD hierdurch nicht und auch die Stimmgewichtung, z. B. auf Parteitagen, wird durch die Onlinethemenforen nicht angetastet. Damit unterscheiden sie sich deutlich von Elementen „,numerischer De- 
mokratie“ (Kersting 2014, S. 61) wie Mitgliederentscheiden, die digital im Stil des „electronic voting“ (Kersting 2014, S. 63) umgesetzt werden könnten.

In Bezug auf etwaige Auswirkungen, welche die hier analysierte Reform mittels der Onlinethemenforen zeitigen können, wird daher vorsichtig umgegangen. Denn die bisher (Stand Juli 2020) einzige, aber manifeste Auswirkung ist die Verankerung der Onlinethemenforen im Parteistatut. Das macht grundsätzlich den Weg frei für eine neue, genuin digitale Parteistruktur. Alle weiteren Auswirkungen sind abzuwarten, auch und besonders im Hinblick auf mögliche Beteiligungszahlen. Denkbar ist allerdings, dass sich durch die neue Gliederungsstruktur eine höhere Mobilisierung junger Neumitglieder ergibt, die aktiv in der Partei mitarbeiten wollen. Dies ist zumindest die Hoffnung der Parteiführung. Sie begründet durch Asynchronität und Ortsungebundenheit der digitalen Technologie einen neuen Kanal des Agendasettings für Mitglieder, die bislang aufgrund räumlicher und zeitlicher Verhinderung nicht in Ortsvereinen oder Arbeitsgemeinschaften mitarbeiten konnten. Die Gruppe der Parteimitglieder, die Möglichkeiten zur Beeinflussung der internen Diskurse haben, aus denen kollektive Entscheidungen hervorgehen, wächst damit um digital organisierte Gruppen. So werden Onlinethemenforen zum parteiinternen Instrument der deliberativen Demokratie und ähneln in ihrer Form digitaler Partizipation in demokratischen Entscheidungssystemen vom Prinzip her elektronischen Bürgerhaushalten (Zobel und Weiß 2018; Kersting 2014, S. 63).

Zudem könnte eine stärkere Bindung neuer Mitglieder erzielt werden, die in den Onlinethemenforen eine Alternative zur Arbeit vor Ort sehen. Für die Partei als Ganzes könnte ein Imagegewinn dergestalt erzielt werden, als dass die SPD als „moderne“ Partei auftritt. Aus den themenorientierten neuen Foren könnten nun Anträge von thematisch höherer Qualität entstehen, weil über die digitale Kollaboration verschiedene Expert*innen mit den Anträgen befasst sind. Möglicherweise könnte sich aus all diesen Faktoren heraus eine neue Art von Parteimitgliedern bilden, deren Parteiidentität sich rund um die Onlinethemenforen aufbaut.

Die auf dem Bundesparteitag 2019 beschlossenen Onlinethemenforen werden ihre Wirkung erst in den kommenden Jahren entfalten. Potenziell bieten sie die Möglichkeit, zu einem neuen Machtzentrum der Partei anzuwachsen. Die Arbeitsgemeinschaft der Jusos hat bei der Wahl der Doppelspitze 2019 eindrucksvoll aufgezeigt, wie zahlenmäßige und diskursive Unterstützung in Entscheidungsprozessen wirkmächtig werden kann. Ob die Onlinethemenforen sich in eine derartige Richtung entwickeln, wird allerdings stark davon abhängen, ob sie große Mitgliederzahlen und hohe Beteiligungsquoten erreichen können, ihre Sprecher*innen zu prominenten Parteipersönlichkeiten werden und sie geschickte Allianzen mit anderen Machtzentren, z.B. in Landesverbänden, schmieden.

Auch ihre technische Form, deren Ausgestaltung nun dem Parteivorstand obliegt, wird darüber bestimmen, ob dort geführte Debatten in kollektiven Kompromissen oder in zerstrittene Handlungsunfähigkeit münden. Die aus der Erfahrung mit der Piratenpartei gespeiste Angst des Scheiterns an komplizierten Sozialbeziehungen in digital organisierten Aushandlungsprozessen gilt als Grenze digitaler Innovation. Elemente wie die Moderation von Diskussionen und kreative Reaktionen auf veränderte Sozialbeziehungen im digitalen Raum sind diesbezüglich von hoher Bedeutung. Denkbar ist auch eine periodische Rückkehr vom digitalen in den analogen 
Raum, z. B. in Form von Jahrestreffen der Foren, die den Mangel an persönlichem Austausch im Internet kompensieren könnten.

\subsection{Reichweite des Ansatzes digitaler Parteireform zur Theoriebildung}

Was bedeuten diese Ergebnisse mit Blick auf die Theoriebildung im Bereich (digitaler) Parteireformen? Grundsätzlich stützen die sechs identifizierten Begründungsmuster die von Gauja (2017) genannten drei Bereiche, die das Feld der Reformbegründungen abdecken (siehe Abschnitt „Gründe für die digitale Organisationsreform 2017-2019“). Allerdings verweisen der Bezug zu sozialen Medien und die gewünschte digitale Vernetzung eindeutig auf die digitale Transformation der Gesellschaft und sind somit vollständig neue Reformgründe, die bislang so nicht beobachtet wurden.

Der kausale Mechanismus, der die Einführung der Onlinethemenforen 2017-2019 innerparteilich zum Beschluss führte, wird als die Verknüpfung legitimatorischer Begründungen und Zielsetzungen verstanden, die alle drei von Gauja (2017) identifizierten Ebenen miteinander verbindet und die legitimatorischen Erwartungen aller relevanten Akteure im Entscheidungsprozess erfüllt. Der kausale Mechanismus ist schließlich eine komplexe Geschichte aus wahrgenommenen Herausforderungen und sinnvollen Lösungen, die der Reforminitiative der Parteiführung über die Umsetzung im institutionalisierten Entscheidungsprozess der Partei Legitimation zur Umsetzung erteilt.

Die spezifische Konstellation, in der die Parteiführung im Anschluss an eine Wahlniederlage als Reforminitiatorin auftrat und die Reforminhalte der digitalen Reform über innerparteiliche Kommissionen so vorbereitete, dass sie auf dem Parteitag als Teil eines größeren Reformpakets beschlossen werden konnten, ist auch in anderen Parteien vorstellbar. Die legitimatorischen Begründungsmuster der Parteiführung gegenüber der Gesamtpartei könnten daher im Sinne von elementaren kausalen Mechanismen als Ansatzpunkt einer verallgemeinerbaren Theorie der Gründe für (digitale) Parteireformen verstanden werden, die an anderen Parteien überprüft werden kann (Nullmeier 2019, S. 168). Allerdings erlaubte der Umfang dieser Studie einen nur eingeschränkten Zugang zu Informationen über interne Verhandlungsrunden. So ist diese Studie eine explorative Annäherung an den innerparteilichen Entscheidungsprozess, der die Sequenz kausaler Mechanismen bis zum Beschluss darstellt. Die Reichweite der Ergebnisse und insofern auch die dementsprechende Theoriebildung ist als erster Schritt eines mehrschrittigen Theoriebildungsprozesses zu verstehen. Den Zusammenhang zwischen spezifischeren Bedingungen, legitimatorisch hervorgebrachten Begründungsmustern als Mechanismen und den Wirkungen der Parteireform herauszuarbeiten, ist somit weiterhin Aufgabe zukünftiger Forschung zu digitalen Parteireformen.

Danksagung Die Autor*innen bedanken sich bei den beiden anonymen Gutachter*innen für ihre konstruktiven Hinweise zur Überarbeitung des Manuskripts, bei Aimie Bouju, Arno von Schuckmann, Stine Ziegler, Anna-Sophie Heinze und dem AK Parteien der DVPW für weitere wichtige Hinweise und bei Maximilian Wilshaus und Tatevik Tophoven-Sedrakyan für die Hilfe bei der Datenaufbereitung. 
Förderung Die Arbeit der Nachwuchsforschungsgruppe DIPART (geleitet von Isabelle Borucki) wird durch das Forschungsprogramm „Digitale Gesellschaft“ des Ministeriums für Kultur und Wissenschaft des Landes Nordrhein-Westfalen finanziert (Fördernummer 005-1709-0003).

Funding Open Access funding provided by Projekt DEAL.

Open Access Dieser Artikel wird unter der Creative Commons Namensnennung 4.0 International Lizenz veröffentlicht, welche die Nutzung, Vervielfältigung, Bearbeitung, Verbreitung und Wiedergabe in jeglichem Medium und Format erlaubt, sofern Sie den/die ursprünglichen Autor(en) und die Quelle ordnungsgemäß nennen, einen Link zur Creative Commons Lizenz beifügen und angeben, ob Änderungen vorgenommen wurden.

Die in diesem Artikel enthaltenen Bilder und sonstiges Drittmaterial unterliegen ebenfalls der genannten Creative Commons Lizenz, sofern sich aus der Abbildungslegende nichts anderes ergibt. Sofern das betreffende Material nicht unter der genannten Creative Commons Lizenz steht und die betreffende Handlung nicht nach gesetzlichen Vorschriften erlaubt ist, ist für die oben aufgeführten Weiterverwendungen des Materials die Einwilligung des jeweiligen Rechteinhabers einzuholen.

Weitere Details zur Lizenz entnehmen Sie bitte der Lizenzinformation auf http://creativecommons.org/ licenses/by/4.0/deed.de.

\section{Literatur}

Altheide, David L., und Robert P. Snow. 1979. Media logic. London, Beverly Hills: SAGE.

Beach, Derek, und Pedersen Rasmus Brun. 2019. Process-tracing methods: foundations and guidelines. Ann Arbor: University of Michigan Press.

Bennett, W. Lance, und Alexandra Segerberg. 2012. The logic of connective action. Digital media and the personalization of contentious politics. Information, Communication \& Society 15(5):739-768.

Berg, Sebastian, Niklas Rakowski, und Thorsten Thiel. 2020. Die digitale Konstellation. Eine Positionsbestimmung. ZPol https://doi.org/10.1007/s41358-020-00207-6.

Bieber, Christoph. 2014. Online-Partizipation in Parteien - Ein Überblick. In Internet und Partizipation. Bottom-up oder Top-down? Politische Beteiligungsmöglichkeiten im Internet, Hrsg. Kathrin Voss, 173-191. Wiesbaden: Springer VS.

Bukow, Sebastian. 2009. Parteiorganisationsreformen zwischen funktionaler Notwendigkeit und institutionellen Erwartungen. In Die Zukunft der Mitgliederpartei, Hrsg. Uwe Jun, Oskar Niedermayer, und Elmar Wiesendahl, 211-228. Opladen: Barbara Budrich.

Bukow, Sebastian. 2013a. Die professionalisierte Mitgliederpartei. Politische Parteien zwischen institutionellen Erwartungen und organisationaler Wirklichkeit. Wiesbaden: Springer VS.

Bukow, Sebastian. 2013b. Die Wiederentdeckung der mitgliedschaftsbasierten Parteiorganisation: Ziele, Prozess und Ergebnisse der SPD-Parteireform 2009-2011. In Abkehr von den Parteien?: Parteiendemokratie und Bürgerprotest, Hrsg. Oskar Niedermayer, Benjamin Höhne, und Uwe Jun, 231-259. Wiesbaden: Springer VS.

Bukow, Sebastian. 2014. Die SPD-Parteiorganisationsreform 2009-2011: Mit Primaries und verstärkter Basisbeteiligung auf dem Weg zur ,modernsten Partei Europas“? In Parteien und Demokratie: Innerparteiliche Demokratie im Wandel, Hrsg. Ursula Münch, Uwe Kranenpohl, und Henrik Gast, 133-150. Baden-Baden: Nomos.

Bukow, Sebastian, und Thomas Poguntke. 2013. Innerparteiliche Organisation und Willensbildung. In Handbuch Parteienforschung, Hrsg. Oskar Niedermayer, 179-209. Wiesbaden: Springer.

Chadwick, Andrew, und Jennifer Stromer-Galley. 2016. Digital media, power, and democracy in parties and election campaigns. The International Journal of Press/Politics 21(3):283-293.

Collier, David. 2011. Understanding process tracing. Political Science and Politics (PS) 44(4):823-830.

Couldry, Nick. 2008. Mediatization or Mediation?: Alternative understandings of the emergent space of digital storytelling. New Media \& Society 10(3):373-391.

de Nève, Dorothée, und Niklas Ferch. 2019. Instrumente innerparteilicher Demokratie. Ein digitales Dossier aus vergleichender Perspektive. Friedrich-Ebert-Stiftung, Abteilung Internationaler Dialog. http://library.fes.de/pdf-files/id/ipa/15887.pdf. Zugegriffen: 12. Apr. 2020. 
Deschouwer, Kris. 1992. The survival of the fittest: measuring and explaining change and adaption of political parties. Paper prepared for presentation at the Workshop on "Democracies and the Organization of Political Parties". Limerick: ECPR.

Diehl, Paula. 2018. Die 5-Sterne-Bewegung als Laboratorium neuer Tendenzen und ihre widersprüchlichen Repräsentationsbeziehungen. In Formwandel der Demokratie, Hrsg. Winfried Thaa, Christian Volk, 127-154. Baden-Baden: Nomos.

Dohle, Marco, Olaf Jandura, und Gerhard Vowe. 2014. Politische Kommunikation in der OnlineWelt. Dimensionen des strukturellen Wandels politischer Kommunikation. Zeitschrift für Politik 61(4):414-436.

Flick, Uwe. 2017. Qualitative Sozialforschung. Eine Einführung, 8. Aufl., Reinbek bei Hamburg: Rowohlt.

Gauja, Anika. 2017. Party reform. The causes, challenges, and consequences of organizational change. Oxford, New York: Oxford University Press.

Gerbaudo, Paolo. 2019. Are digital parties more democratic than traditional parties? Evaluating podemos and movimento 5 Stelle's online decision-making platforms. Party Politics https://doi.org/10.1177/ 1354068819884878.

Gerl, Katharina, Stefan Marschall, und Nadja Wilker. 2016. Innerparteiliche Demokratie 2.0? Zeitschrift für Vergleichende Politikwissenschaft 10(S2):115-149.

Gläser, Jochen, und Grit Laudel. 2010. Experteninterviews und qualitative Inhaltsanalyse als Instrumente rekonstruktiver Untersuchungen. Wiesbaden: Springer VS.

Hanel, Katharina, und Stefan Marschall. 2014. Der Einsatz von Online-Tools durch Parteien - Am Beispiel von onlineantrag.spd.de. In Internet und Partizipation, Hrsg. Kathrin Voss, 193-201. Wiesbaden: Springer VS.

Harmel, Robert, und Kenneth Janda. 1994. An integrated theory of party goals and party change. Journal of Theoretical Politics 6(3):259-287.

Helfferich, Cornelia. 2014. Leitfaden- und Experteninterviews. In Handbuch Methoden der empirischen Sozialforschung, Hrsg. Nina Baur, Jörg Blasius, 559-574. Wiesbaden: Springer VS.

Jun, Uwe. 2009. Organisationsreformen der Mitgliederparteien ohne durchschlagenden Erfolg: Die innerparteilichen Veränderungen von CDU und SPD seit den 1990er Jahren. In Die Zukunft der Mitgliederpartei, Hrsg. Uwe Jun, Oskar Niedermayer, und Elmar Wiesendahl, 187-210. Opladen: Barbara Budrich.

Jun, Uwe. 2018. Direkte innerparteiliche Demokratie in der parlamentarischen Demokratie: Das Beispiel der Mitgliederpartei SPD. Zeitschrift für Parlamentsfragen 49(4):940-950.

Kersting, Norbert. 2014. Online Beteiligung - Elektronische Partizipation - Qualitätskriterien aus Sicht der Politik. In Internet und Partizipation, Hrsg. Kathrin Voss, 53-87. Wiesbaden: Springer VS.

Klecha, Stephan, und Alexander Hensel. 2013. Zwischen digitalem Aufbruch und analogem Absturz: Die Piratenpartei. Opladen, Berlin, Toronto: Barbara Budrich.

Korte, Karl-Rudolf, und Jan Treibel (Hrsg.). 2012. Wie entscheiden Parteien? Prozesse innerparteilicher Willensbildung in Deutschland. Baden-Baden: Nomos.

Korte, Karl-Rudolf, Dennis Michels, Jan Schoofs, Niko Switek, und Kristina Weissenbach. 2018. Parteiendemokratie in Bewegung. Organisations- und Entscheidungsmuster der deutschen Parteien im Vergleich. Die politischen Parteien der Bundesrepublik Deutschland. Baden-Baden: Nomos.

Koschmieder, Carsten. 2016. Partizipation in der Piratenpartei. Die Schattenseiten einer sonnigen Utopie. Opladen: Barbara Budrich.

Kruse, Jan. 2015. Grundlagentexte Methoden, 2. Aufl., Qualitative Interviewforschung. Ein integrativer Ansatz. Weinheim, Basel: Beltz Juventa.

Marschall, Stefan. 2013. „Mitgliederpartei 2.0“. Chancen und Grenzen virtueller Parteimitgliedschaft. In Parteien ohne Mitglieder?: Tagungsband „Parteien ohne Mitglieder? “. Düsseldorf, 23. bis 24. Oktober 2009., Hrsg. Ulrich von Alemann, Martin Morlok, und Tim Spier, 271-287. Baden-Baden: Nomos.

Mergel, Ines, Noella Edelmann, und Nathalie Haug. 2019. Defining digital transformation: results from expert interviews. Government Information Quarterly 36(4):1-16.

Merkens, Hans. 2017. Auswahlverfahren, Sampling, Fallkonstruktion. In Qualitative Forschung. Ein Handbuch, 12. Aufl., Hrsg. Uwe Flick, Ernst von Kardorff, und Ines Steinke, 286-299. Reinbek bei Hamburg: Rowohlt.

Meuser, Michael, und Ulrike Nagel. 2009. Das Experteninterview - konzeptionelle Grundlagen und methodische Anlage. In Methoden der vergleichenden Politik- und Sozialwissenschaft: Neue Entwicklungen und Anwendungen, Hrsg. Susanne Pickel, Gert Pickel, Hans-Joachim Lauth, und Detlef Jahn, 465-479. Wiesbaden: VS. 
Niedermayer, Oskar. 2019. Parteimitgliedschaften im Jahre 2018. Zeitschrift für Parlamentsfragen 50(2):385-410.

Nullmeier, Frank. 2019. Interpretative Politikforschung und kausale Mechanismen. Zeitschrift für Politikwissenschaft 29(2):153-171.

Panebianco, Angelo. 1988. Political parties. Organization and power. Cambridge studies in modern political economies. Cambridge: Cambridge University Press.

Saldaña, Johnny. 2009. The coding manual for qualitative researchers. Los Angeles: SAGE.

Schmidt, Jan-Hinrik. 2009. Das neue Netz. Merkmale, Praktiken und Folgen des Web 2.0. Konstanz: UVK.

Schwartz-Shea, Peregrine, und Dvora Yanow. 2013. Interpretive research design. Concepts and processes. New York: Routledge.

SPD-Bundesparteitag (Hrsg.). 2019. Organisationspolitische Neuaufstellung. Beschluss Nr. 2 vom 6. Dezember 2019. https://indieneuezeit.spd.de/fileadmin/pv/Dokumente/BPT2019/Beschluesse/B2_ Beschluss_Organisationspolitische_Neuaufstellung.pdf. Zugegriffen: 18. Dez. 2019.

SPD-Parteivorstand (Hrsg.). 2017. Die \#SPDerneuern: Unser Weg nach vorn. Beschluss vom 27. November 2017. https://www.spd.de/fileadmin/Dokumente/Bundesparteitag_2017/Ordentlicher_BPT/ 20171127_LA_Arbeitsprogramm.pdf. Zugegriffen: 15. Jan. 2019.

Tenscher, Jens, und Isabelle Borucki. 2015. Politische und mediale Logiken. Ein Vorschlag zur Differenzierung politischer Medialisierung. In Wandel und Kontinuität der Politischen Kommunikation, Hrsg. Michael Jäckel, Uwe Jun, 139-168. Opladen: Barbara Budrich.

Treibel, Jan. 2012. Was bedeutet innerparteiliche Willensbildung? In Wie entscheiden Parteien? Prozesse innerparteilicher Willensbildung in Deutschland, Hrsg. Karl-Rudolf Korte, Jan Treibel, 7-34. BadenBaden: Nomos.

Treibel, Jan. 2013. Innerparteiliche Entscheidungsprozesse: Interne Machtverhältnisse und Modi der Entscheidungsfindung. In Handbuch Regierungsforschung, Hrsg. Karl-Rudolf Korte, Timo Grunden, 359-369. Wiesbaden: Springer VS.

Vorwärts. 2018. Nur für Mitglieder: SPD startet Debattenportal zur Erneuerung. Vorwärts Webseite vom 26. September 2018. https://www.vorwaerts.de/artikel/nur-mitglieder-spd-startet-debattenportalerneuerung. Zugegriffen: 15. Jan. 2019.

Walter-Rogg, Melanie. 2013. Parteireformen und ihre Wirkung auf die Mitgliederentwicklung. In Parteien ohne Mitglieder?, Hrsg. Ulrich von Alemann, Martin Morlok, und Tim Spier, 247-271. BadenBaden: Nomos.

Wiesendahl, Elmar. 2010a. Der Organisationswandel politischer Parteien: Organisations- und wandlungstheoretische Grundlagen. In Parteien in Theorie und Empirie Parteien als fragmentierte Organisationen: Erfolgsbedingungen und Veränderungsprozesse, Bd. 1, Hrsg. Uwe Jun, Benjamin Höhne, 35-61. Opladen: Barbara Budrich.

Wiesendahl, Elmar. 2010b. Zwei Dekaden Party Change-Forschung: Eine kritische Bilanz. In Krise oder Wandel der Parteiendemokratie?: Festschrift für Ulrich von Alemann, Hrsg. David Gehne, Tim Spier, 92-118. Wiesbaden: VS.

Zobel, Maria-Stephanie, und Jens Weiß. 2018. Wirkung von E-Partizipation: Erkenntnisse aus Evaluationen elektronischer Bürgerhaushalte. In Wirkungen von E-Government, Hrsg. Jürgen Stember, Wolfgang Eixelsberger, und Andreas Spichiger, 207-245. Wiesbaden: Springer. 